\title{
Control theory of metabolic channelling
}

\author{
Boris N. Kholodenko, ${ }^{1}$ Marta Cascante $^{2}$ and Hans V. Westerhoff ${ }^{3,4}$ \\ ${ }^{1}$ A.N. Belozersky Institute of Physico-Chemical Biology, Moscow State University, 119899 Moscow, Russia; \\ ${ }^{2}$ Department de Bioquimica i Fisiologia, Universitat de Barcelona, Marti i Franques 1, Barcelona, Spain; \\ ${ }^{3}$ E.C. Slater Institute, University of Amsterdam, Plantage Muidergracht 12; ${ }^{4}$ Department of Molecular Biology, H5, The \\ Netherlands Cancer Institute, Plesmanlaan 121, NL 1066 CX, The Netherlands
}

\begin{abstract}
Various factors appear to control muscle energetics, often in conjunction. This calls for a quantitative approach of the type provided by Metabolic Control Analysis for intermediary metabolism and mitochondrial oxidative phosphorylation. To the extent that direct transfer of high energy phosphates and spatial organization plays a role in muscle energetics however, the standard Metabolic Control Theory does not apply, neither do its theorems regarding control.

This paper develops the Control Theory that does apply to the muscle system. It shows that direct transfer of high energy phosphates bestows a system with enhanced control: the sum of the control exerted by the participating enzymes on the flux of free energy from the mitochondrial matrix to the actinomyosin may well exceed the $100 \%$ mandatory for ideal metabolic pathways. It is also shown how sequestration of high energy phosphates may allow for negative control on pathway flux. The new control theory gives methods functionally to diagnose the extent to which channelling and metabolite sequestration occur. (Mol Cell Biochem 143: 151-168, 1995)
\end{abstract}

Key words: metabolic channeling, control analysis, muscle energy metabolism, metabolite/enzyme sequestration

\section{Introduction}

Quantitative approaches have led to appreciable advances in understanding the control of cellular metabolism. In the framework of these approaches the intuitive concept of ratelimiting step has been substituted by the more subtle definition of control exerted by any enzyme on the flux. The quantitative formulation was introduced by Higgins [1] and, in more elaborated form, by Kacser and Burns [2, 3], Savageau [4] and Heinrich and Rapoport [5, 6]. It was renamed to flux control coefficient of an enzyme by Burns $e t$ al. [7] in the context of metabolic control analysis.

Treating cellular metabolism as a network of enzyme reactions control analysis used to be based on the following important assumptions: (1) the rate of any reaction is proportional to the concentration of enzyme (a property often termed additivity [8]), (2) different enzymes are independent catalysts, (3) the concentrations of enzyme-bound metabolites can be neglected as compared to their free concentrations (sub- stitution of the latter by the total metabolite concentrations greatly simplifies control analysis $[9,10])$.

These assumptions, which are true for simple or 'ideal' [11] metabolic pathways result in the equivalence of different ways of defining and measuring the control coefficients of enzymes [12-15]. Moreover, they allow one to describe metabolic processes at the 'macroscopic' level of 'block' (i.e., the enzyme) reactions without going to the details of reaction mechanisms. With these assumptions great advances were achieved in derivation and interpretation of the control coefficients of enzymes, and, moreover, the ('global') pathway control properties have been expressed in terms of the ('local') regulatory properties of the individual enzyme reactions and of the structure of a pathway [14-18].

However, cellular metabolism can not be always reduced to a number of independent enzyme reactions occurring in a well-stirred continuous tank reactor (for which the assumptions of the classical theory hold true). In highly organized cellular metabolic pathways direct enzyme-enzyme interac-

Address for offprints: B.N. Kholodenko, A.N. Belozersky Institute of Physico-Chemical Biology, Moscow State University, 119899 Moscow, Russia *This article was published in Molecular and Cellular Biochemistry 133/134: 313-331, 1994. Kluwer Academic Publishers regret the publication of the uncorrected version. 
tions and enzyme associations take place (for reviews see [19-22]). They lead to a restricted diffusion or/and even to a direct transfer of metabolites between interacting enzymes $[19,23,24]$. In some pathways such direct transfer is even standard, for example, in parts of the electron transfer chains of free energy transducing membranes and in the bacterial phosphotransferase systems for sugar uptake [25].

Membrane linked free energy transduction involving proton pumping [26] has also been proposed to involve direct transfer of protons or associated electric fields between adjacent proton pumps (see [27] as a review). Also in this case such transfer would normally occur in parallel to the generation of a proton electrochemical potential difference between the two aqueous bulk phases bordering the energy coupling membrane. Consequently, conclusive evidence for such proton channelling is still rare.

More recently, it has been shown that a somewhat neglected structural feature of mitochondria, i.e., the outer mitochondrial membrane, warrants greater interest. Molecules such as adenine nucleotides permeate this membrane through a pore to which hexokinase may bind. Inner and outer mitochondrial membranes are in close contact at certain sites. In the context of channelling of high-Gibbs-energy phosphates the issue has been raised of the relative location of adenine nucleotide translocase, creatine kinase, adenylate kinase, hexokinase, contact site and porin [28-32].

A number of authors have extended the control analysis approach to special cases of the regulation of systems with enzyme-enzyme interaction and metabolic channelling (see, e.g. $[8,33-36])$. However, no control theory dealing with the general case of partial channelling, dynamic and/or static, has been developed. Following the work [11] we here develop the essentials of such a control theory for a pathway with metabolic channelling at steady state.

\section{Quantitative definitions of control}

The loose question whether the particular enzyme $i$ does or does not control the flux can be made both operational and quantitative by rephrasing it as follows: If we change the activity of the enzyme $i$ by, say, $1 \%$ what will be the percentage change in that flux at the steady state? If there is no variation in the flux, clearly this enzyme does not control the flux at all. In case the flux also changes by $1 \%$ the enzyme $i$ may be called rate-limiting: the flux completely follows the activity of that enzyme. A strict mathematical definition extrapolates the percent variation to an infinitesimal one and relates a fractional change $(\mathrm{d} J / J)$ in the steady-state flux to the fractional modulation $\left(\mathrm{d} e_{i} / e_{i}\right)$ of the enzyme concentration [3]:

$$
C_{e_{i}}^{J}=\left(\frac{\mathrm{d} J / J}{\mathrm{~d} e_{i} / e_{i}}\right)_{s y s}=\left(\frac{\mathrm{d} \ln |J|}{\mathrm{d} \ln e_{i}}\right)_{s y s}
$$

The subscript sys signifies that differentiation conditions require the steady state of the system and allow the concentrations of metabolites to adjust accordingly.

The dimensionless coefficient $C_{e_{i}}^{J}$ is called the flux control coefficient of the enzyme $i$ [7]. As was mentioned, if the control coefficient equals zero, the enzyme $(i)$ is non-rate-limiting and in case it equals unity the enzyme is rate-limiting. Thus, these two categories of the older qualitative analysis are retained in the new terminology. More importantly, however, in any intermediate case the control coefficient can take any value and it can be considered as a quantitative indicator of the relative degree to which the enzyme controls (or limits) the overall flux.

In ordinary (ideal) metabolic pathways there is a one-toone correspondence between the enzymes and reactions, and another definition for the control coefficient $[5,6]$ compares a relative change of the local rate $\left(\delta v_{i} / v_{i}\right)$ through a reaction $i$ with a variation $(\delta J / J)$ of the system's (steady-state) flux caused by a change in the rate $v_{i}$. The control coefficient defined in this manner is designated by $C_{v_{i}}^{\prime}$ [8], and is referred to as the control coefficient with respect to the activity or rate of an enzyme to emphasize its relation to a 'local' rate, $v_{i}$. A short-hand notation $[5,6,7]$ for this definition reads:

$$
C_{v_{i}}^{j}=\frac{(d J / J)_{s y s}}{\left(\partial v_{i} / v_{i}\right)_{e n z}}
$$

The subscriptenz signifies that a perturbation in the local rate $\left(\partial v_{i} / v_{i}\right)$ is considered as if the enzyme $i$ reaction were in isolation from the pathway (therefore, only the $i$-th reaction is required to be at steady state). The subscript sys signifies that after an initial perturbation of the local rate the pathway as a whole attains a new steady state. The mathematically more explicit expression considers changes in an enzyme rate as caused by a variation in any parameter $p_{i}$ which affects only the rate $v_{i},\left(\partial v_{i} / \partial p_{i} \neq 0, \partial v_{j} / \partial p_{i}=0\right.$ for any $\left.j \neq i\right)$ :

$$
C_{v_{i}}^{J}=\frac{\left(\mathrm{d} \ln |J| / \mathrm{d} p_{i}\right)_{s y s}}{\left(\partial \ln \left|v_{i}\right| / \partial p_{i}\right)_{e n z}}=\frac{v_{i}}{J} \frac{\left(\mathrm{d} J / \mathrm{d} p_{i}\right)_{s y s}}{\left(\partial v_{i} / \partial p_{i}\right)_{e n z}}
$$

(When taking the derivative $\partial v_{i} / \partial p_{i}$ all the concentrations of metabolites should be kept at the values of the initial steady state of the pathway).

In an analogous manner (see Eqs 1-3) the concentration control coefficients can be defined. They measure the response of the concentration of a particular metabolite to changes in a specific parameter (e.g., $e_{i}$ or $p_{i}$ ):

$$
\begin{aligned}
C_{e_{i}}^{x_{k}} & =\left(\frac{d x_{k} / x_{k}}{d e_{i} / e_{i}}\right)_{s y s}=\left(\frac{\mathrm{d} \ln x_{k}}{\mathrm{~d} \ln e_{i}}\right)_{s y s} \\
C_{v_{i}}^{x_{k}} & =\frac{\left(\mathrm{d} \ln \mid x_{k} / \mathrm{d} p_{i}\right)_{s y s}}{\left(\partial \ln \left|v_{i}\right| / \partial p_{i}\right)_{e n z}}=\frac{v_{i}}{J} \cdot \frac{\left(\mathrm{d} x_{k} / \mathrm{d} p_{i}\right)_{s y s}}{\left(\partial v_{i} / \partial p_{i}\right)_{e n z}},
\end{aligned}
$$


where $x_{k}$ is the concentration of a metabolite. For lack of space we shall mainly deal with the control of the fluxes through a system.

In ideal pathways the definitions of the control coefficients with respect to the enzyme rate do not depend on the choice of a parameter $p_{i}[12,14,15,37]$. Considering in Eq. 3 the enzyme concentration $\left(e_{i}\right)$ as a parameter $\left(p_{i}\right)$ one can see, that in ideal pathways (where a reaction rate is proportional to the enzyme concentration, $v_{i} \alpha e_{i}$, see Introduction) the control coefficient with respect to the rate $\left(C_{v_{i}}^{J}\right)$ is completely equivalent to that one with respect to the enzyme concentration $\left(C_{e}^{j}\right)$. However, in non-ideal pathways, for example, in metabolic pathways with high enzyme concentrations and moiety conservations, the coefficients $C_{v_{i}}^{J}$ and $C_{e_{i}}^{J}$ may differ significantly $([9,10]$, see also Section 3$)$. Moreover, the control coefficient in a non-ideal pathway, as defined according to Eq. 3 (or Eq. $5)$, may depend on the choice of parameter $p_{i}([10,38]$, see an example in Section 5). This dependence appears due to direct or indirect (i.e., via the moiety conservation involving different enzymes bound to the same substrate moiety) interactions between enzymes. Indeed, below we shall see that the difference in the control coefficients can be used to diagnose channelling (see also [34, 36, 38]).

One of the best known and intuitively most understandable properties of the flux control coefficients is that when summing over all enzymes of a system they add up to unity:

$$
\sum_{i} C_{e_{i}}^{J}=1
$$

In ideal pathways this property, called the summation theo$\mathrm{rem}$, is valid independently of the structure of a pathway and the local kinetic properties of the enzymes. Indeed, in such pathways any steady-state flux is a homogeneous first order function of the enzyme concentrations (i.e., if the enzyme concentrations simultaneously change by a factor $(\alpha)$, all fluxes in the system will change by the same factor $(\alpha)$, and the summation theorem (Eq. 6) is a simple consequence of Euler's theorem (cf. $[15,39,40]$ ). However, if a metabolic pathway is non-ideal, the sum in Eq. 6 may be not equal to unity. The behavior of the sum of the flux control coefficients in different systems will be analyzed below. We shall see that measuring this sum can give a deeper insight into regulatory properties of the real cellular pathways.

\section{Static and dynamic channelling: partly channelled pathways}

In the traditional view enzymes in an aqueous solution obtain their substrates from a well-stirred bulk phase and return their products back to that phase. As was mentioned in the
Introduction the existence of alternative forms of metabolic organization including enzyme-enzyme interactions and enzyme associations requires to extend this naive concept. The term 'channelling' has been used by a number of authors to indicate a sequence of chemical conversions taking place within enzyme complexes without releasing the intermediate metabolites into the bulk phase. The reaction sequence in such a 'channelled' phase may be catalyzed by various types of enzyme associations. In the case of so called 'static' channelling the enzymes in the complex are tightly bound, i.e. such complexes exist longer than the mean passage time of metabolites through that part of the pathway. In an extreme case different monomers can be linked covalently as, for instance, in the pyruvate dehydrogenase and tryptophan synthase enzyme complexes [41]. Obviously, in the latter case the concentrations of different 'domains' of the complex can not be varied independently, so that the question how variations of the concentrations of different enzymes (domains) affect the flux becomes inoperational. Much of the current debate in the literature is more concerned with weak enzyme associations, often requiring the presence of the common intermediate [42-45]. In this case the amount of complex enzymes depends dynamically on the metabolite concentrations and the flux through that part of the pathway. If such an association of enzymes leads to a direct transfer of an intermediary metabolite, it is referred to as 'dynamic channel'. In the following we develop a general control theoretical treatment applicable to an arbitrary physico-chemical mechanism of channelling (static or dynamic, different schemes that are possible in general are described in detail in $[45,46])$.

For a moment we return to a simple metabolic pathway in which channelling is absent (Fig. 1). Traditionally this pathway is treated in terms of two consecutive, enzyme catalyzed reactions without going to the detailed enzyme mechanisms. Each of the reactions, e.g., reaction 1 , has a control coefficient over the pathway flux defined by considering a small change in the total concentration of enzyme $\left(e_{1}\right)$, see Eq. 1. Here we note, that in the framework of a 'microdescription' (see Fig. 1) such an increase of $e_{1}$ corresponds to a simultaneous proportional increase in all the forward and reverse (pseudo-) first order rate constants of the enzyme 1. To make this statement exact, we need to define the control coefficients of the elemental processes (steps) of the enzyme 1 with respect to the overall steady-state flux through the pathway $\left(C_{i}^{J}\right)$ :

$$
C_{i}^{\prime}=\left(\mathrm{d} \ln |J| / \mathrm{d} \ln k_{i}^{-}\right)_{s y s}, k_{i}^{-} / k_{i}^{+}=\text {const, } i=1,2
$$

where the differentiation conditions are such that the forward $\left(k_{i}^{+}\right)$and the reverse $\left(k_{i}^{-}\right)$rate constants of the elemental process $i$ are changed by the same factor, all other parameters being kept constant. It should be noted that this definition [cf., 


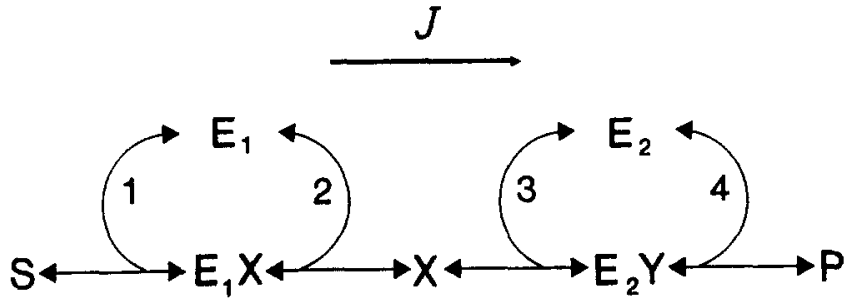

Fig. 1. 'Ideal' pathway of two enzymes. The concentrations of the initial substrate, $S$, and the end product, $P$, are constants. $X$ is the intermediate in the bulk phase. Steps 1,2 and 3,4 are $E_{1}$ - and $E_{2}$-dependent elemental processes, respectively. The positive direction of the flux $J$ (from the substrate $S$ to the product $P$ ) is indicated.

$47,48]$ does not affect microscopic reversibility. An equivalent, but more general definition of the elemental control coefficient $\left(C_{i}^{J}\right)$ can be given in a manner similar to Eq. 3. In this case, however, the rate $\left(v_{j}\right)$ becomes the rate of the $i^{\text {th }}$ elemental process rather than the rate of the entire $i$ reaction. Most importantly, when addressing the elemental processes Eq. 3 defines the control coefficient, $C_{i}^{J}$, as general quantity, independent of a special choice of a parameter $p_{i}$ for both ideal and non-ideal cellular pathways [Kholodenko et al., submitted]:

$$
C_{i}^{J}=\frac{\left(\mathrm{d} \ln |J| / \mathrm{d} p_{i}\right)_{s y s}}{\left(\partial \ln \left|v_{i}\right| / \partial p_{i}\right)_{p r o c}}
$$

The analogous definition for the elemental control coefficient over any concentration $x_{k}$ reads:

$$
C_{i}^{x_{k}}=\frac{\left(\mathrm{d} \ln x_{k} / \mathrm{d} p_{i}\right)_{s y s}}{\left(\partial \ln \left|v_{i}\right| / \partial p_{i}\right)_{\text {proc }}}
$$

Now, for the pathway of Fig. 1, the control coefficients relative to both the concentration $\left(C_{e_{1}}^{J}\right)$ or the activity $\left(C_{v e_{1}}^{J}\right)$ of the enzyme 1 can be written as:

$$
C_{e_{1}}^{J}=C_{v e_{1}}^{J}=C_{1}^{J}+C_{2}^{J}
$$

Here the symbol $v e_{1}$ is used to emphasize that the control coefficient is related to the entire enzyme $e_{1}$ rate rather than to the rate of the elemental step.

For the pathway of Fig. 1 the summation theorem (Eq. 6) can be written as:

$$
C_{e_{1}}^{J}+C_{e_{2}}^{J}=C_{1}^{J}+C_{2}^{J}+C_{3}^{J}+C_{4}^{J}=1
$$

This reformulation of the summation theorem for simple pathways may be useful for the cases where one is attempting to understand what the implication is of a regulation of one of the elemental steps in an enzyme catalyzed reaction on the flux through the metabolic pathway. However, here we merely use it as a prelude to the control theoretical treatment for the channelled systems in Fig. 2. Figure 2a repre- sents the example of a static channel which was analyzed by Sauro and Kacser [33]. It was assumed that the enzymes form the complex $\left(\mathrm{Q}=E_{1} E_{2}\right)$ independently of their interactions with metabolite molecules. Complex $Q$ catalyzes the direct conversion of $\mathrm{S}$ to $\mathrm{P}$, denoted as the step 5 in Fig. $2 \mathrm{a}$. In this such a static channel, the number of enzyme molecules involved in the channel does not vary with the metabolic flux. Figure $2 b$ denotes the more general case of dynamic channelling, where the extent of channelling depends on the relative rates of collision of $E_{1} X$ and $E_{2}$ compared to the rate of dissociation of $E_{1} X$ into $E_{1}$ plus $X$ and also on the other rate constants. The reaction sequence in the 'channelled phase' corresponds to steps 5 and 6 and is represented by the lower route in Fig. 2b.

Figure 2 may illustrate the problem of defining control coefficients of the participating enzymes in the case of channelling. For instance, enzyme 1 participates in two rather than one reaction (the channelled reaction from $S$ to $P$ as well as the reaction from $\mathrm{S}$ to $\mathrm{X}$ ). Indeed, this is where classical control theory got stuck. Sauro and Kacser [33] were the first who for the case of static channelling (Fig. 2a) have indicated a solution. Assuming thermodynamic equilibrium between the separate enzymes and the complex they calculated the elasticities of partial reactions (i.e., proceeding in the bulk phase and in the channelled phase) with respect to the total enzyme concentrations. However, only under special conditions these elasticities which are referred to as protein $(\pi)$ elasticities $[8,33]$ depend only on the equilibrium constant and the total amounts of the enzymes. This approximation fails for the more general case of a dynamic channel of Fig. 2b. Moreover, one should take care applying this method to a static channel if association/dissociation of enzyme monomers can no longer be assumed significantly slower than the catalytic reactions.

Figure 3 shows a pathway which possesses features of a static channel. At any steady state of the system the step 5 of the complex formation is at thermodynamic equilibrium. However, if this step is not significantly slower than other steps and if the concentrations of the enzyme forms with bound ligands are comparable to the concentrations of the corresponding free enzyme forms, the $\pi$ elasticities of partial reactions should depend on the flux and the concentrations of intermediates and can not be calculated only in terms of the equilibrium constant and the total amount of the enzymes. For, to fulfil the conditions necessary for the calculation of the $\pi$ elasticities via equilibrium parameters, one would have to assume that the affinity of $E_{1}$ for $E_{2}$ does not depend upon whether a ligand $(X$ or $Y)$ has bound. This is not consistent with a scheme of Fig. 3, already because it requires the existence of a complex $E_{1} X E_{2} Y$. It can be shown, that the correct determination of the $\pi$ elasticities requires an analysis at the level of the elemental processes of a system [Kholodenko et $a l$, in preparation]. 

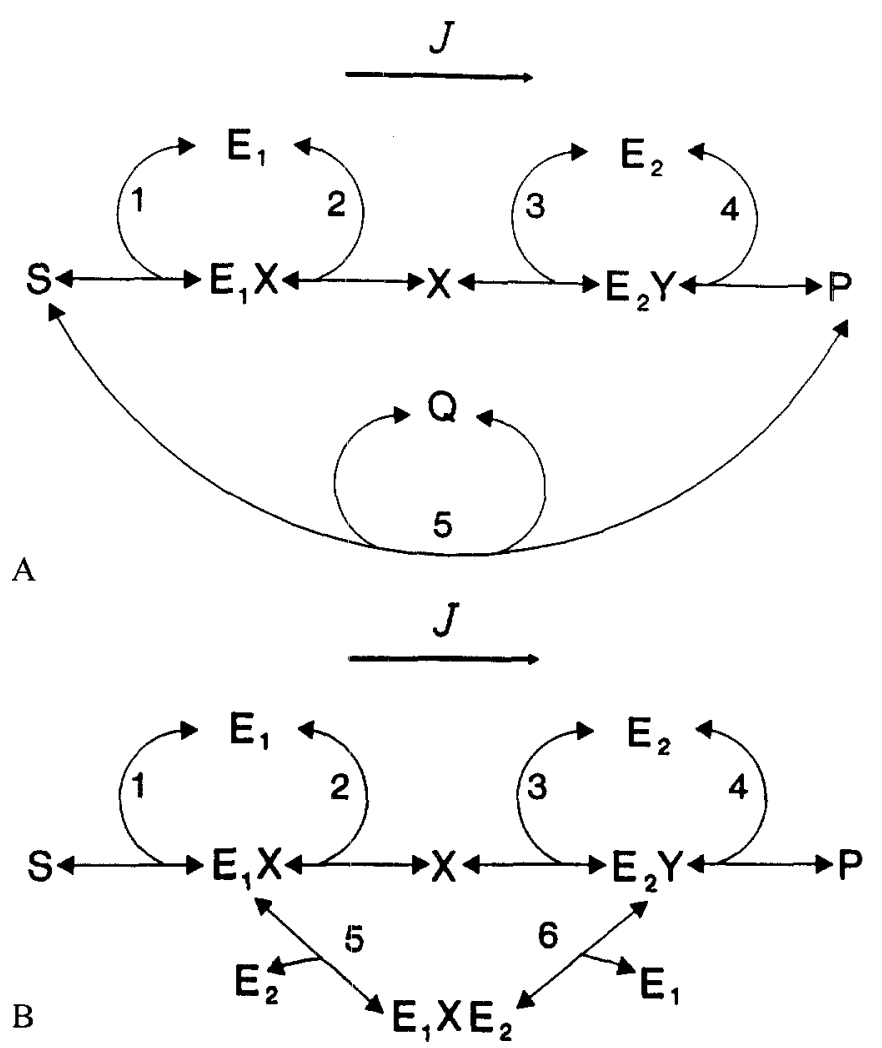

Fig. 2. 'Static' (a) and 'dynamic' (b) channels. The dynamic complex $E_{1} X E_{2}$ is formed only after binding $X$ to $E_{1}$, while the static complex $Q$ is formed independently of the presence of a common intermediate. In either system the upper route represents the usual reaction pathways through the bulk phase intermediate $X$, catalyzed by free enzymes, and the lower routes represent the 'channelling'. (a): steps 1,2,5 and 3, 4,5 are $E_{1}$ - and $E_{2}$-dependent processes, respectively. (b): steps $1,2,5,6$ and $3,4,5,6$ are $E_{1}$ - and $E_{2}$-dependent processes, respectively.

Our consideration of Fig. 1 above now suggests a solution to this dilemma: one should recognize that Figs 2a, $2 \mathrm{~b}$ and 3 are still networks of chemical conversions and that they may be treated in terms of control coefficients with respect to the elemental processes (Eq. 8). These processes correspond to transitions between different states of enzymes, or to sequences of such transitions that are not interrupted by branches. Consequently, there are five, six and eight elemental flux control coefficients for the systems in Figs $2 a, 2 b$ and 3 , respectively. For example, for Fig. 2b:

$$
C_{6}^{J}=\partial \ln |J| / \partial \ln k_{6}^{+} \text {, where } k_{6}^{-} / k_{6}^{+}=\text {const, }
$$

Because the flux through the system is a homogeneous function of the elemental rate constants the following summation theorem holds for this channelled system [cf., 39]:

$$
C_{1}^{J}+C_{2}^{J}+C_{3}^{J}+C_{4}^{J}+C_{5}^{J}+C_{6}^{J}=1,
$$

i.e., the sum of the flux control coefficients continues to equal 1 provided the sum is over all the elemental processes.

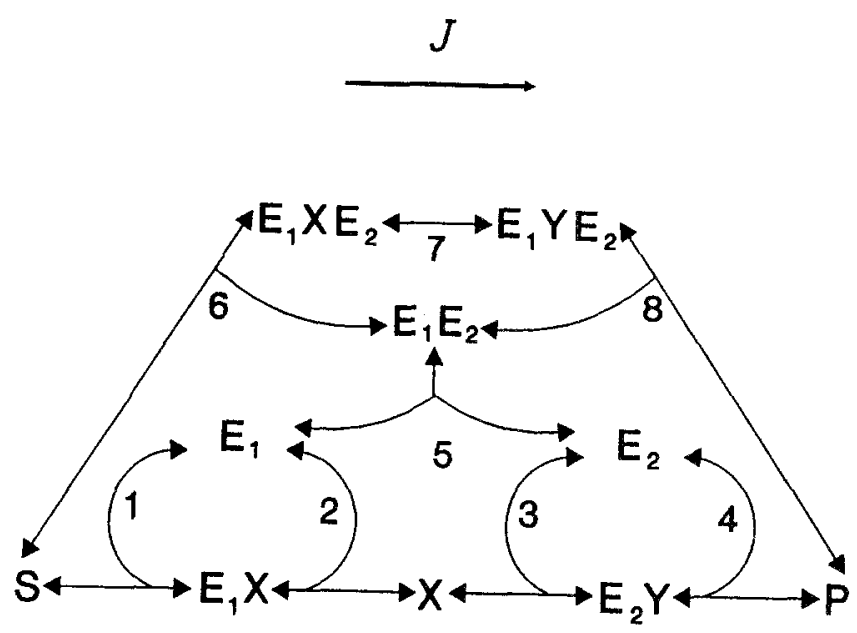

Fig. 3. 'Static' channel where only the free ('empty') forms of the enzymes 1 and $2, E_{1}$ and $E_{2}$, can associate into the complex.

Now from the level of the elemental processes of a pathway (the 'microdescription' level) we return to the level of enzyme reactions. The concept of elemental control coefficients allows us to assign an analogue of the control coefficient with respect to the enzyme activity $\left(C_{v_{i}}^{J}\right)$ to the individual enzymes of a channel. Suppose, we simultaneously change the elemental rate constants of all processes in which the enzyme $i$ is involved, by the same factor. Considering the corresponding change in the steady state flux $J$ we define the 'impact' control coefficient, ${ }^{\mathrm{imp}} C_{e_{i}}^{J}$, as the sum of the elemental control coefficients over the processes in which any of the forms of the enzyme $i$ is involved ( $E_{i}$-dependent processes):

$$
{ }^{\operatorname{imp}} C_{e_{i}}^{J}=\underset{\substack{\text { all } E_{i} \text {-dependent } \\ \text { processes } k}}{\sum} C_{k}^{J}
$$

This coefficient evaluates the total impact enzyme $i$ has on the flux $J$ (the term ' $E_{i}$-dependent' refers to any form of enzyme $i$, monomeric or complexed, that contains $E_{i}$-moiety). In Sections 4 and 5 we explain this terminology more thoroughly by considering the responses of channelled pathways to signal molecules and the experimental methods of measuring the control coefficients (see also $[38,47]$ ). Here we note that 'channelled' elemental steps are the $E_{i}$ - and $E_{j}$-dependent processes simultaneously when enzymes $i$ and $j$ form a complex involved in catalytic transformations. In fact, for the scheme of Fig. 2a:

$$
{ }^{\mathrm{imp}} C_{e_{1}}^{J}=C_{1}^{J}+C_{2}^{J}+C_{5}^{J}, \quad{ }^{\mathrm{imp}} C_{e_{2}}^{J}=C_{3}^{J}+C_{4}^{J}+C_{5}^{J}
$$

Consequently, the channelled step 5 contributes to the control exerted by either enzyme. For the scheme of Fig. $2 b$ :

$$
{ }^{\mathrm{imp}} C_{e_{1}}^{J}=C_{1}^{J}+C_{2}^{J}+C_{5}^{J}+C_{6}^{J}
$$




$$
{ }^{\mathrm{imp}} C_{e_{2}}^{J}=C_{3}^{J}+C_{4}^{J}+C_{5}^{J}+C_{6}^{J}
$$

We note that, the definition of the impact control coefficient ${ }^{i m p} C_{e_{1}}^{J}$ (by modulation of activities of all $E_{1}$-dependent processes) does not correspond to just a change in the total concentration of the enzyme 1 at constant distribution of this enzyme over all its forms and at a constant concentration of the enzyme 2 . Indeed, the concomitant change in the form $E_{1} X E_{2}$ violates the conservation constraint imposed on the total concentration of the enzyme 2. So, in cases of enzymeenzyme interactions as well as in other non-ideal pathways there is a difference between the control coefficients defined in terms of modulations of activity and those defined in terms of modulations of the enzyme concentration (cf. Eqs 1, 2 and 11). Both definitions are important since they refer to different experimental methods of determining the control coefficients (see Section 5). Here, for the channelled pathways of Fig. 2 we relate these two types of the control coefficients (see Section 7 for the corresponding equations and their derivation for the general case):

$$
\begin{aligned}
& C_{e_{1}}^{J}+C_{e_{2}}^{J} \cdot e_{\mathrm{i} 2}^{\mathrm{comp} / e_{2}}={ }^{\mathrm{imp}} C_{e_{1}}^{J} \\
& C_{e_{1}}^{J} \cdot e_{\mathrm{1} 2}^{\mathrm{comp}} / e_{1}+C_{e_{2}}^{J}={ }^{\mathrm{imp}} C_{e_{2}}^{J}
\end{aligned}
$$

Here $e_{12}^{\text {comp }}=\left[E_{1} E_{2}\right]$ or $\left[E_{1} X E_{2}\right]$ for the static (Fig. 2a) or the dynamic (Fig. 2a) channel, respectively, $e_{1}$ and $e_{2}$ are the total concentrations of the enzymes. In view of Eqs 15 or 16 one can use Eq. 17 to express the control coefficients with respect to enzyme concentrations into the control coefficients of the elemental processes. This then allows one also to evaluate the expected magnitude of the sum of the enzyme control coefficients. For the dynamic channel the result reads:

$$
C_{e_{1}}^{j}+C_{e_{2}}^{y}=\left(1+C_{5}^{j}+C_{6}^{j}\right) /\left(1+e_{12}^{\text {comp } / e}\right)
$$

For simplicity we here considered the case where the total concentrations of the two enzymes are equal $(e)$. Considering $J$ as well as the overall flux through the pathway the relative contribution of the channelled steps to flux control can be expressed as following (see the branch theorems in [15, 16]):

$$
\left(C_{5}^{J}+C_{6}^{J}\right) / J_{\text {chan }}=\left(C_{2}^{J}+C_{3}^{J}\right) /\left(J-J_{\text {chan }}\right)
$$

where $J_{\text {chan }}$ denotes the flux through the channel. Using Eq. 13 one arrives at:

$$
C_{e_{1}}^{J}+C_{e_{2}}^{J}=\left\{1+\left(J_{\text {chan }} / J\right) \cdot\left(1-\left(C_{1}^{J}+C_{4}^{J}\right)\right)\right\} /\left(1+e_{12}^{\text {comp } / e}\right)
$$

In the case of static channelling (Fig. $2 \mathrm{a}$ ) $C_{1}^{J}$ and $C_{4}^{J}$ disappear from this expression. Eq. 19 shows that the magnitude of the sum of the flux control coefficients can vary from less than unity to two depending on the ratio of the channelled and bulk-phase fluxes and the kinetic properties of the enzymes involved.

\section{Responses of channelled pathways to regulatory signals}

External effectors often play a role as signal molecules causing the system to modify its behavior in order to meet altered environmental requirements. In order to understand the cells regulatory structure it is important to realize how the response of the whole system is related to the 'local' response of the reactions directly affected by the signallers. By analyzing the responses to signals we shall arrive at the methods of experimental diagnosis of channelling (see Sections 5 and 6 ). We now show that the response to signals of 'channelled' pathways can differ drastically from the response of the corresponding non-channelled pathways.

The response of the steady-state flux $(J)$ toward an external signal (effector) is quantified by the response coefficient $\left(R_{\sigma}^{J}\right)$, defined as $\log$ - $\log$ derivative of the flux with respect to the concentration of 'signal' molecules $(\sigma)$ (cf. Eq. 1) [3]:

$$
R_{\sigma}^{J}=\left(\frac{\mathrm{d} J / J}{\mathrm{~d} \sigma / \sigma}\right)_{s y s}=\left(\frac{\mathrm{d} \ln |J|}{\mathrm{d} \ln \sigma}\right)_{s y s}
$$

In ideal pathways a metabolic response to a signal is determined by the flux control coefficient and by the elasticity coefficient $\left(\varepsilon_{\sigma_{i}}^{e_{i}}\right)$ of the rate of the receptor ('target') enzyme (i) with respect to this signal [3]:

$$
R_{\sigma_{i}}^{J}=\left(\frac{\mathrm{d} \ln |J|}{\mathrm{d} \ln e_{i}}\right)_{s y s} \cdot\left(\frac{\partial \ln \left|v_{i}\right|}{\partial \ln \sigma_{i}}\right)_{e n z}=C_{c_{i}}^{J} \cdot \varepsilon_{\sigma_{i}}^{c_{i}}
$$

This relationship requires that signal molecules $\sigma_{i}$ affect only one enzyme $i$ in the pathway, as denoted by the subscript $i$.

In metabolic pathways with enzyme-enzyme interactions Eq. 21 is no longer valid. One of the reasons is lack of a oneto-one correspondence between enzymes and independent reactions. Now, one can guess that to derive a general expression for the responses of non-ideal pathways one may venture to the level of the elemental processes, i.e., use the 'microdescription' of a pathway. The elasticity coefficient of the elemental process $k$ with respect to the signal molecules present at concentration $\sigma_{i}$ will be designated as,

$$
\varepsilon_{\sigma_{i}}^{k}=\left(\partial \ln \left|v_{k}\right| / \partial \ln \sigma_{i}\right)_{p r o c}
$$

Then, one can find the response of the flux to a signal specific to its receptor enzyme $(i)$ via the control coefficients $\left(C_{k}^{J}\right)$ of the $E_{i}$-dependent elemental processes $\left(v_{k}\right)$ and their elasticities $\left(\varepsilon_{\sigma_{i}}^{k}\right)$, (the response theorem [49]): 


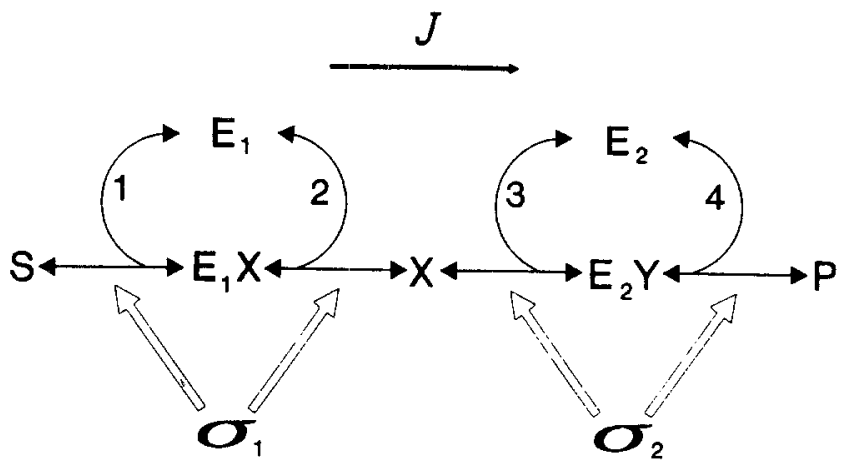

Fig. 4. How signal molecules affect an ideal pathway. $\sigma_{1}$ and $\sigma_{2}$ are the signal molecules, specific to the receptor enzymes 1 and 2 , respectively. For other details see the legend to Fig. 1.

$$
R_{\sigma_{i}}^{J}=\sum_{\substack{\text { all } E_{i} \text {-dependent } \\ \text { processes k }}} C_{k}^{J} \cdot \varepsilon_{\sigma_{i}}^{k}
$$

As was mentioned above, some of the elemental processes may depend on two enzymes simultaneously, if these enzymes form a complex involved in catalytic transformations.

Applying Eq. 22 to the schemes of Figs 4 and 5 one can see, that additional terms appear in the response of channelled pathways to signallers. For example, the flux responses to a signal $\left(\sigma_{1}\right)$ affecting the enzyme 1 in the simple (non-channelled) pathway (Fig. 4) and in the 'static' channel (Fig. 5a) are, respectively:

$$
R_{\sigma_{1}}^{J}=C_{1}^{J} \cdot \varepsilon_{\sigma_{1}}^{1}+C_{2}^{J} \cdot \varepsilon_{\sigma_{1}}^{2}=C_{e_{1}}^{J} \cdot \varepsilon_{\sigma_{1}}^{e_{1}}
$$

(the superscript $e_{1}$ denotes the elasticity of the entire enzyme rate with respect to signal molecules), and

$$
R_{\sigma_{1}}^{J}=C_{1}^{J} \cdot \varepsilon_{\sigma_{1}}^{1}+C_{2}^{J} \cdot \varepsilon_{\sigma_{1}}^{2}+C_{5}^{J} \cdot \varepsilon_{\sigma_{1}}^{5}
$$

Obviously, the term $C_{5}^{J} \cdot \varepsilon_{\sigma_{1}}^{5}$ is absent in the response of the pathway of two unchannelled enzyme reactions, Eq. [23]. In the response $\left(\mathrm{R}_{\sigma_{2}}^{J}\right)$ of the channelled flux to a signal $\sigma_{2}$ affecting the enzyme 2 an analogous additional term $\left(C_{5}^{J} \cdot \varepsilon_{\sigma_{2}}^{5}\right)$ appears.

The flux responses to signaller for the 'dynamic' channel (Fig. 5b) are:

$$
\begin{aligned}
& R_{\sigma_{1}}^{J}=C_{1}^{J} \cdot \varepsilon_{\sigma_{1}}^{1}+C_{2}^{J} \cdot \varepsilon_{\sigma_{1}}^{2}+C_{5}^{J} \cdot \varepsilon_{\sigma_{1}}^{5}+C_{6}^{J} \cdot \varepsilon_{\sigma_{1}}^{6} \\
& R_{\sigma_{2}}^{J}=C_{3}^{J} \cdot \varepsilon_{\sigma_{2}}^{3}+C_{4}^{J} \cdot \varepsilon_{\sigma_{2}}^{4}+C_{5}^{J} \cdot \varepsilon_{\sigma_{2}}^{5}+C_{6}^{J} \cdot \varepsilon_{\sigma_{2}}^{6}
\end{aligned}
$$

Eqs 23-25 already suggest that the response of channelled pathways to signals differs from that of non-channelled ones. To compare the responses it is convenient to normalize them to the response of the receptor enzyme by itself. In the light
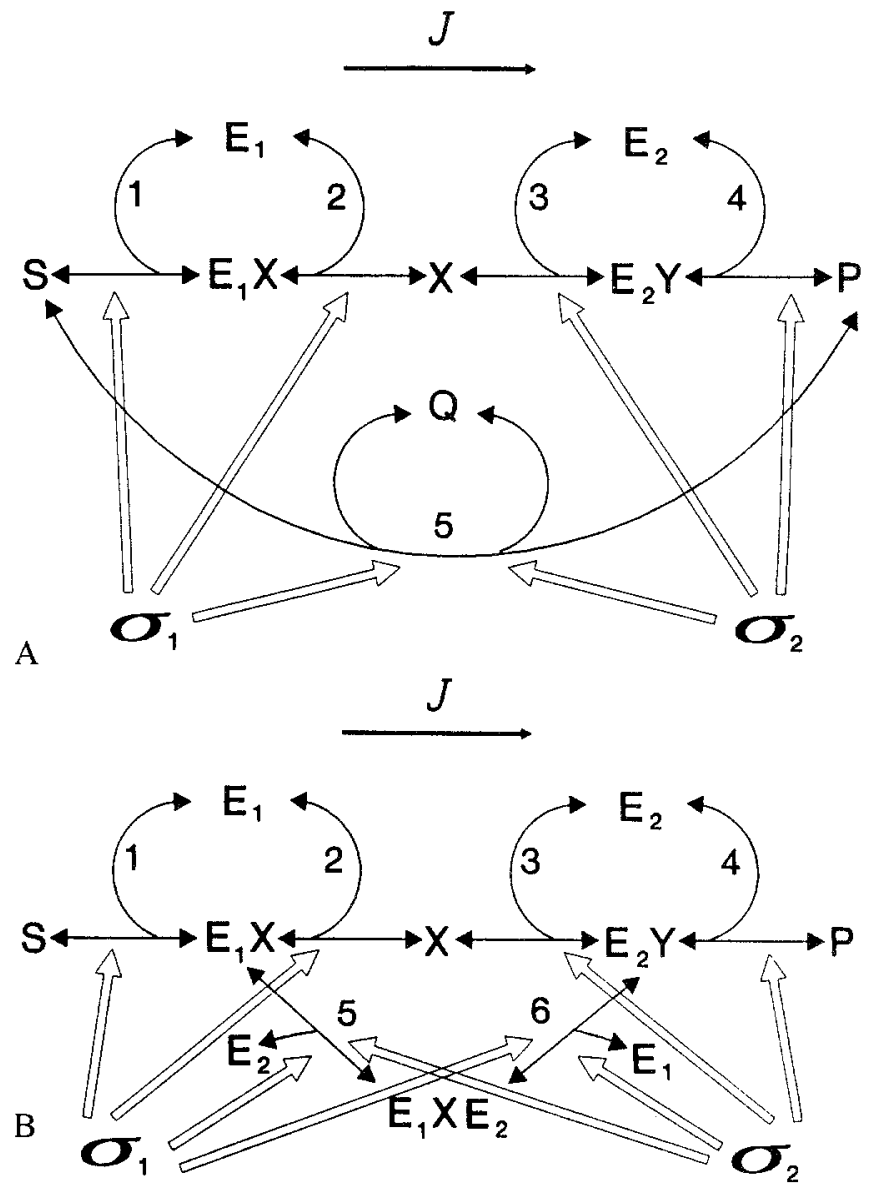

Fig. 5. How signal molecules affect 'channelled' pathways. (a): 'static' and (b) 'dynamic' channels. $\sigma_{1}$ and $\sigma_{2}$ are the signal molecules, specific to the receptor enzymes 1 and 2 , respectively. Other details see in the legend to Fig. 2.

of definitions of the control theory (see Eqs 3 and 20), this normalized response will be called the 'signal transduction' coefficient of the enzyme $i,{ }^{R} C_{e_{i}}^{J}\left(\sigma_{i}\right)$. This signal transduction coefficient is equal to the ratio of the response of the whole pathway to the response of the 'isolated' enzyme:

$$
{ }^{R} C_{e_{i}}^{J}\left(\sigma_{i}\right)=R_{\sigma_{i}}^{J} / \varepsilon_{\sigma_{i}}^{e_{i}}=\sum_{\substack{\text { all } E_{i} \text {-dependent } \\ \text { processes } k}} C_{k}^{J} \cdot \varepsilon_{\sigma_{i}}^{k} / \varepsilon_{\sigma_{i}}^{e e_{i}}
$$

here the superscript $e_{i}$ denotes the elasticity of the rate of the enzyme $i$ considered as if in isolation from the pathway. In ideal systems the signal transduction coefficient coincides with the classical control coefficient of the enzyme (see Eq. 23). In non-ideal pathways the value of the former may depend not only on the pathway properties but also on the peculiarities of signal molecules, as emphasized by the argument $\sigma_{i}$ of the signal transduction coefficient.

Here we will consider the simple case when the elasticities 


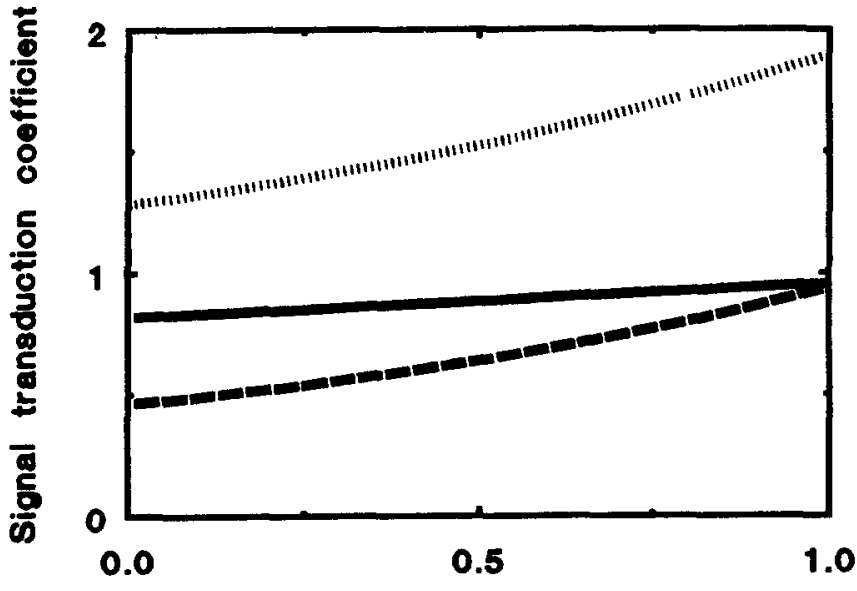

channelled flux fraction

Fig. 6. Channelling enhances response to a signal. Signal transduction coefficients for enzyme 1 (solid line) and 2 (dashed line) of Fig. $5 \mathrm{~b}$ and their sum (dotted line) were calculated as functions of the fraction of the total flux running through the channel. Leakage of bulk phase $(X)$ and of channelled $\left(E_{1} X E_{2}\right)$ intermediates was added to the scheme shown in Fig. $5 \mathrm{~b}$. Total concentrations of enzymes 1 and 2 were set to 1 . Elemental rate constants were equal to: $k_{1}^{+}=10, k_{1}^{-}=0,1 k_{2}^{-}=10 \alpha, k_{2}^{-}=\alpha, k_{3}^{+}=\alpha, k_{3}^{-}=\alpha$, $k_{4}^{+}=10, k_{4}^{-}=0.01, k_{5}^{+}=100 \beta, k_{5}^{-}=\beta, k_{6}^{+}=0.0045 \beta, k_{6}^{-}=0.045 \beta$, and for 'channelled' (subscript $l c$ ) and 'unchannelled' (subscript $I$ ) leaks considered as irreversible steps, $k_{k}=2.316 \cdot 10^{3} \cdot \beta, k_{l}=0.05 \alpha . \alpha$ and $\beta$ were chosen such that the total output flux $\left(J_{\text {oul }}\right.$, to product $\left.\mathrm{P}\right)$ and the total leak flux remained constant (at 0.3869 and $0.323 J_{o u t}$, respectively). The channelled flux fraction was defined as the flux from $E_{1} X E_{2}$ to $E_{2} Y$ divided by $J_{\text {ou }}$ (in the case considered it also equals to the channelled flux before the leak reaction divided by $J_{\text {in }}$. The signal transduction and control coefficients were calculated by increasing elemental rate constants $\left(k_{1}^{+}, k_{1}^{-}, k_{2}^{+}, k_{2}^{-}, k_{5}^{+}, k_{5}^{-}, k_{6}^{+}, k_{6}^{-}, k_{l c}\right)$ for enzyme $E_{1}$ and $k_{3}^{+}, k_{3}^{-}, k_{4}^{+}, k_{4}^{-}, k_{5}^{+}, k_{9}^{-} k_{6}^{+}$, $k_{6}^{-}, k_{l c}$ ) for enzyme $E_{2}$ by $0.01 \%$.

$\left(\varepsilon_{\sigma_{i}}^{k}\right)$ of all $E_{i}$-dependent elemental processes to the signal are equal to each other and to the elasticity of the reaction catalyzed by the enzyme $i$ in 'isolation' $\left(\varepsilon_{\sigma_{i}}^{e_{i}}\right)$ :

$$
\varepsilon_{\sigma_{i}}^{k}=\varepsilon_{\sigma_{i}}^{e_{i}} \text {, if } k \text { is an } E_{i} \text {-dependent process }
$$

It follows from Eqs 26 and 27 that in this case the expression for the signal transduction coefficient will be following:

$$
{ }^{R} C_{e_{i}}^{J}\left(\sigma_{i}\right)=\sum_{\substack{\text { all } E_{i} \text {-dependent } \\ \text { processes } k}} C_{k}^{J}={ }^{i m p} C_{e_{i}}^{J}
$$

Thus, for signals satisfying Eq. (27), the signal transduction coefficient coincides with the impact control coefficient (Section 3). Accordingly, the enzyme then transduces such signals via all the processes in which any of its forms is involved, and the contribution of every process is determined only by its flux control coefficient.
It is worth mentioning, that treating in this manner some inhibitors as signals, one may indicate a way of measuring the control coefficients (see Section 5). Here, for signals satisfying Eq. (27) we compare the signal transduction coefficients of enzymes in ideal and channelled pathways, see Figs 4 and 5 , respectively. It follows from the equations above (see Eqs 23-28), that for either enzyme the same additional term is present in the channelled pathway equating,

$$
C_{5}^{J}=J_{\text {chan }} / J
$$

for the static channel (Fig. 5a) or,

$$
C_{5}^{J}+C_{6}^{J}=\left(J_{\text {chan }} / J\right) \cdot\left(1-\left(C_{1}^{J}+C_{4}^{J}\right)\right)
$$

for the dynamic channel (Fig. 5b). In case a significant fraction $J_{\text {chan }} / J$ of the flux flows through the channel, the signal transduction coefficient of each of the enzymes 1 and 2 can be close to unity, whereas only one of them can have such a value in the pathway of two unchannelled enzyme reactions.

In realistic cases the intermediates may be subject to leakage. In such a case the input flux ( $J_{i n}$, the substrate consumption) differs from the output flux ( $J_{\text {ou }}$, synthesis of the product), the difference equals the sum of the leaks of bulk phase $(X)$ and channelled $\left(E_{1} X E_{2}\right)$ intermediates. Figure 6 shows how the signals can control the output flux at various degrees of channelling in the case when 'channelled' and 'unchannelled' leaks are proportional to the corresponding 'channelled' and 'unchannelled' fluxes. We can see that the signal transduction coefficient of either enzyme $\left({ }^{R} C_{e_{i}}^{\left.J_{\text {out }}\right)}\right.$ increases in parallel with the fraction of the flux that runs through the channel.

In a simple case only the bulk phase intermediate is subject to leakage. Then, the signal transduction coefficient $\left({ }^{R} C_{e_{i}}^{J_{\text {out }}}\right)$ of either enzyme includes an additional term which in the case of the static channel is equal to:

$$
C_{5}^{\text {out }}=J_{\text {chan }} / J_{\text {out }}
$$

for the dynamic channel,

$$
C_{5}^{J_{\text {out }}}+C_{6}^{J} \text { out }=\left(J_{\text {chan }} / J_{\text {out }}\right) \cdot\left(1-C_{4}^{J}\right)-\left(J_{\text {chan }} / J_{\text {in }}\right) \cdot C_{1}^{J}
$$

Under the condition that most of the bulk-phase flux ends up in the leak and the binding of $\mathrm{S}$ and $\mathrm{P}$ to $E_{1}$ and $E_{2}$, respectively, are near equilibrium, the signal transduction coefficient of each of the enzymes $E_{1}$ and $E_{2}$ should be close to unity even if the channelled flux is much smaller than total bulk phase flux but comparable to the output flux. We conclude that channelling tends to increase the response of a pathway to signals. 


\section{Experimental methods for determining the control coefficients}

Classical definition of the enzyme's control coefficient, Eq. 1 , has the operational implication of modulating the concentration of that enzyme. However, direct titration of the amount of an enzyme is possible only in a reconstituted system [50]. In native systems a change in the concentration of the enzyme of interest can be achieved by genetic means, for instance by comparing heterocaryons of Neurospora crassa [51], by causing a gene to be expressed from a plasmid [52], or by modulating the expression of the chromosomal gene [53]. This approach has the complication that the modulation of a single gene may result (although non-directly) in changes of the concentrations of several enzymes. On the other hand, Eq. 3 allows one to measure the control coefficients both in intact and reconstituted systems using inhibitors or other modificators specific to single enzymes [54-56].

In ideal pathways, the result of such a measurement does not depend on the type of inhibitor used, provided that the inhibitor $\left(I_{i}\right)$ only affects the target enzyme $(i)$. However, this is not true for non-ideal systems, for instance with direct enzyme-enzyme interactions. To emphasize this we called the coefficients, determined according to classical theory, the 'apparent' control coefficients and denoted them by a left upper index 'app' (cf. Eq. 26) [38, 47]:

$$
{ }^{a p p} C_{e_{i}}^{J}\left(I_{i}\right)=\frac{\left(d \ln |J| / d I_{i}\right)_{s y s}}{\left(\partial \ln \left|v_{i}\right| \partial I_{i}\right)_{e n z}}=\frac{v_{i}}{J} \cdot \frac{\left(d J / d I_{i}\right)_{s y s}}{\left(\partial v_{i} / \partial I_{i}\right)_{e n z}}
$$

Here the elasticity of the rate $\left(v_{i}\right)$ of the 'target' enzyme to the inhibitor is considered as if the enzyme $i$ were in isolation from the pathway at the same metabolite concentrations as in the steady state of the system,

$$
\varepsilon_{\sigma_{i}}^{e_{i}}=\left(\partial \ln \left|v_{i}\right| / \partial I_{i}\right)_{e n z}
$$

Since usually the inhibitor, $I_{i}$, is absent in the intact system, all the derivatives in Eq. 29 should be calculated at $I_{i}=0$. Therefore, we used the derivatives with respect to $I_{i}$ rather than to $\ln I_{i}$, in order to avoid indefiniteness connected with the logarithmic derivatives at zero inhibitor concentration (the usual definition of the elasticity coefficients in the control analysis uses logarithmic derivatives, cf. Eq. 21). For instance, if $I_{i}$ is an irreversible inhibitor binding to the enzyme $i$ in the stoichiometry ratio 1:1, Eq. 29 takes the form [54]:

$$
{ }^{a p p} C_{e_{i}}^{J}\left(I_{i}\right)=-\frac{\left(I_{\text {end }}\right)}{J} \cdot \frac{\partial J}{\left.\partial I_{i}\right|_{I_{i}}=0}
$$

Here $I_{\text {end }}$ denotes the inhibitor concentration necessary to completely suppress the enzyme (in the titration curve $I_{\text {end }}$ is determined as a concentration extrapolating to where flux $J$ no longer depends on $\left.I_{i}\right), \partial J / \partial I_{\mid I=0}$ is determined by the initial slope of the titration curve.

We start with a simple numerical example showing that the result of an experiment on titrating a channelled pathway with inhibitor does depend on the type of that inhibitor even if the latter affects only a single enzyme [47, Kholodenko et al., submitted]. We consider a simple dynamic channel (Fig. 2b), and suppose that in order to measure the control coefficient of enzyme 1 on the total flux through the pathway (i.e. the net production rate of $P$ ) one uses different specific inhibitors of the enzyme: (i) a purely non-competitive inhibitor $\left(I_{1}^{m}\right)$ that binds to all the forms of the enzyme 1 independently of their interactions with ligand or enzyme 2 molecules and (ii) a purely competitive inhibitor $\left(I_{1}^{c}\right)$ that binds only to the free form, $E_{1}$, which is not complexed with any ligand or enzyme 2. The corresponding formulas for the calculation of the control coefficients using purely non-competitive and purely competitive inhibitors $\left(I_{1}^{n c}\right.$ and $I_{1}^{c}$ ) read (see, e.g., [54]):

$$
\begin{aligned}
& { }^{a p p} C_{e_{1}}^{J}\left(I_{1}^{n c}\right)=-\frac{\left(K_{1}^{n c}\right)}{J} \cdot \frac{\partial J}{\partial I_{1}^{n c} \mid I_{1}^{n c}=0} \\
& { }^{a p p} C_{e_{1}}^{J}\left(I_{1}^{c}\right)=-\frac{\left(K_{1}^{c}\right)}{J} \cdot\left(1+\frac{S}{K_{M}^{s}}+\frac{X}{K_{M}^{X}}\right) \cdot \frac{\partial J}{\partial I_{1}^{c} \mid I_{1}^{c}=0}
\end{aligned}
$$

Here $K_{1}^{n c}$ and $K_{1}^{c}$ are the inhibition constants of the inhibitors $I_{1}^{n c}$ and $I_{1}^{c}$, respectively. (To ensure the validity of Eqs 32 and 33 the values of the inhibition constants should exceed significantly the total concentration of enzymes, or $I$ should be interpreted as the free inhibitor concentration [47].) $K_{M}^{S}$ and $K_{M}^{X}$ are Michaelis constants for the substrate $(S)$ and product $(X)$ of the enzyme 1, respectively.

The titration curve (the dependence of the steady-state flux $(J)$ on the inhibitor concentration) was simulated by solving the equations determining the steady state of the pathway of Fig. $2 \mathrm{~b}$ for the following set of parameters (in dimensionless units): all the elemental rate constants equaled 1 , except for $k_{1}=k_{4}^{-}=0.5$; the total concentrations of enzymes were taken $e_{1}=e_{2}=1 ; K_{1}^{n c}=10, K_{1}^{c}=10, S=10, \mathrm{P}=1$. Then, Eqs 32 and 33 were applied. The apparent control coefficient of the enzyme 1 on the flux converged to 0.51 when determined using the purely noncompetitive inhibitor $\left(I_{1}^{n c}\right)$ and to 0.40 when determined by use of the purely competitive inhibitor $\left(I_{1}^{c}\right)$. Clearly, contrary to the classical results, the flux control coefficient in this channelled system depends on how it is determined.

A way how to determine the apparent control coefficients in the general case of channelling was outlined in Section 4. We can consider molecules of an inhibitor $\left(I_{i}\right)$ as signal molecules affecting only enzyme $i$. Then, in view of Eq. 26, the formula for the apparent control coefficient, Eq. 29, takes the form: 


$$
{ }^{a p p} C_{e_{i}}^{J}\left(I_{i}\right)=\sum_{\substack{\text { all } E_{i} \text {-dependent } \\ \text { processes } k}} C_{k}^{J} \cdot \varepsilon_{l_{i}}^{k} / \varepsilon_{l_{i}}^{e_{i}}
$$

Here the elasticity coefficients $\left(\varepsilon_{1}^{k}\right)$ of the elemental processes with respect to the inhibitor are defined as:

$$
\varepsilon_{e_{i}}^{v^{k}}=\left(\partial \ln \left|v_{k}\right| / \partial I_{l}\right)_{p m c}
$$

(similarly as above the derivatives with respect to $I_{i}$ rather than to $\ln I_{i}$ are used here in order to avoid indefiniteness at zero inhibitor concentration).

There are various types of inhibitors and various mechanisms of inhibition (see, e.g., [57, 58]). For pathways with enzyme-enzyme interactions this variety may even be greater since the response of the pathway flux to the inhibitor titration strongly depends on how that inhibition affects the enzyme-enzyme complexes. For instance, in the traditional case a non-competitive inhibitor is thought of as an inhibitor that does not replace metabolites at their binding sites in the enzyme molecule. A purely non-competitive inhibitor is usually considered to affect only the parameter $V_{\max }$ of the steady-state rate equation, which in the framework of "microdescription' corresponds to a simultaneous proportional decrease in all the forward and reverse (pseudo-) first order rate constants of the affected enzyme (cf., [57]). However, the addition of such an inhibitor specific to the enzyme $i$ to a channelled pathway results in an equal relative inhibition of the activities of all $E_{i}$-dependent elemental processes only if the following conditions are fulfilled: (i) this inhibitor binds to all the enzyme $i$ forms with the same binding constant, (ii) its binding to any enzyme $i$ form transforms the latter into a less active (or inactive) state, (iii) its binding does not change the ability of the enzyme $i$ to form complexes with the other enzymes of the pathway. In such cases the elasticities of all the $E_{i}$-dependent elemental processes are equal to each other and to the elasticity of the reaction catalyzed by the enzyme $i$ in 'isolation' (cf. Eq. 28):

$$
\varepsilon_{i}^{k n}=\varepsilon_{I_{i}^{n c}}^{l_{i}} \text { if } k \text { is an } E_{i} \text {-dependent process }
$$

where the symbol $I_{i}^{n c}$ denotes a purely non-competitive inhibitor (cf. the numerical example above). It follows from Eqs 34 and 36 that titrating the system with such an inhibitor we measure the impact control coefficient of the enzyme $i$ :

$$
{ }^{a p p} C_{e_{i}}^{J}\left(I_{i}^{n c}\right)={ }^{\mathrm{imp}} C_{e_{i}}^{J}
$$

This control coefficient can not be measured by a direct variation in the enzyme concentration, e.g., by modulating the gene encoding the enzyme. Importantly, the impact control coefficient can differ drastically from the control coefficient with respect to the enzyme concentration (see Fig. 6, where
Eq. 28 is assumed to be fulfilled).

A competitive inhibitor competes with a substrate (product) for the binding sites at the enzyme molecule. In terms of a 'mechanistic' model the molecules of such an inhibitor displace the substrate molecules from the binding site. A purely competitive inhibitor is thought to affect only the parameters $\mathrm{K}_{\mathrm{m}}-\mathrm{s}$ of the steady-state rate equation (cf., [57]). Generalizing this definition one can consider such a purely competitive inhibitor $\left(I_{i}^{c}\right)$ of the enzyme $i$ that binds only to the form, $E_{i}$, which is not complexed with any other metabolite or enzyme of a pathway. Such an inhibitor will suppress the rates of only those elemental processes in which the 'empty' form, $E_{i}$, is a 'substrate' or 'product'. Hence, in a channelled system Eq. 36 is not valid for a purely competitive inhibitor $\left(I_{i}^{c}\right)$ of the enzyme $i$, and the control coefficient of the enzyme i measured with $I_{i}^{c}$ will differ of that of determined by use of Eq. 37 and a purely non-competitive inhibitor $I_{i}^{n c}$. Indeed, we have witnessed a difference between these apparent control coefficients in the numerical example above. Such a difference becomes especially notable in the cases of very tight-binding and irreversible (covalently bound) inhibitors to which Eq. 31 can be applied. It can be shown, that titrating a channelled pathway with an irreversible purely competitive inhibitor one determines (by virtue of Eq. 31) the classical control coefficient $\left(C_{e^{J}}\right)$ with respect to the enzyme concentration, whereas titrating that pathway with irreversible purely non-competitive inhibitor (and using Eq. 31) one measures the impact control coefficient ${ }^{(i m p} C_{e_{i}}^{\prime}$ ) of the enzyme $i$ [Kholodenko and Westerhoff, submitted].

Thus, one may conclude that the finding the different flux control coefficients for the same enzyme using different inhibitors may be an indication that this enzyme is involved in a partially channelled pathway. Another such indication can be any difference between the sum of enzyme control coefficients and unity. A more thorough analysis of the problem can be found in the next Section.

\section{Implementation of control theory to the experimental diagnosis of channelling}

We saw already (see Section 3, Eq. 19) that when a direct transfer of the intermediate takes place, the sum of flux control coefficients $\left(C_{e_{i}}^{J}\right)$ over all enzymes generally differs from unity. The contrary is not true, however [33]. In fact, suppose that enzyme association merely leads to a change in kinetics of the constituent enzymes, and channelling of the intermediate does not occur, Fig. 7. In this case there is only a bulk phase pool of the intermediate $(X)$, and all reaction pathways include diffusional steps to (from) $X$. However, since the 


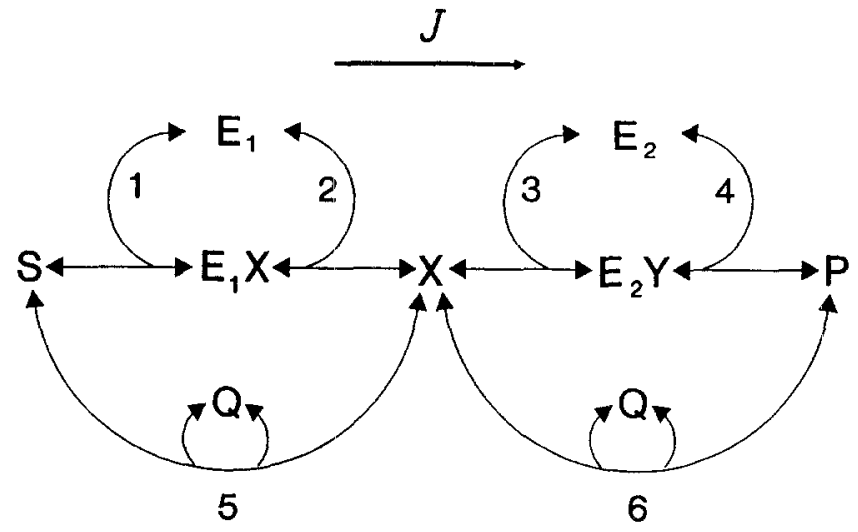

Fig. 7. Pathway of two enzymes capable to form a complex (Q), where channelling is absent. In this system there is only a bulk phase pool of the intermediate $(X)$, and all reaction pathways include diffusional steps to and from $X$.

ratios between concentrations of the free enzymes and the complex changes with a change in the total enzyme concentration, also in this case, the sum of the flux control coefficients will differ from unity for this system (as well as for the systems with channelling shown in Fig. 2).

Therefore, the sum of the classical control coefficients $\left(C_{e}^{J}\right)$ of the enzymes may not be used as the sole criterion for discriminating between direct transfer and a free diffusion mechanism. In the particular case of equal concentrations of both enzymes, Sauro \& Kacser [33] proposed to distinguish between channelling and free diffusion by following the bulk phase intermediate $X$. When the total enzyme concentration, $e_{1}+e_{2}$, is varied the concentration of $X$ does change in the system shown in Fig. 7 (in which the direct transfer is absent), whereas it does not change in the case of a static channel (Fig. 2a).

However, this criterion fails in systems with dynamic complexes. As was mentioned, in this case an enzyme complex is formed only if one of the enzymes has already bound the common intermediate, Fig. $2 \mathrm{~b}$. It has been shown, that even if the total concentrations of both enzymes are equal, the concentration of the bulk phase intermediate $X$ will change upon a change in the total enzyme concentration $e_{1}+e_{2}[47]$.

Our consideration of the responses of the flux to signal (Fig. 6) or inhibitor molecules (Section 5) already suggests that the sum of the signal transduction control coefficients (apparent control coefficients in the case of inhibitor titration) seemed to be useful for discrimination between channelling and a free diffusion mechanism. The question arises: what is this sum equal to for the system shown in Fig. 7?

We shall now consider titrations of pathways with enzymeenzyme complexes and a direct (Fig. 2) or a free diffusion mechanism (Fig. 7) of metabolite transfer with purely noncompetitive inhibitors. For channelled pathways, upon binding with the complex $E_{1} E_{2}$, an inhibitor specific to enzyme 1 may inhibit channelling. Similarly a second inhibitor, binding specifically to 2 may do the same (see Fig. 5 with the signal molecules $\sigma_{1}$ and $\sigma_{2}$ considered now as inhibitors specific to the enzyme 1 and 2, respectively). We saw that such an inhibition of channelling by either inhibitor made the apparent control coefficient of both enzyme 1 and enzyme 2 include the elemental control coefficients of the shared 'channelled' steps (when Eq. 36 is fulfilled). On the other hand, in the case of a free diffusion mechanism of metabolite transfer (Fig. 7), binding of an inhibitor specific for enzyme 1, to the complex $E_{1} E_{2}$, may affect the catalytic steps in the reaction catalyzed by the enzyme 1 (which is bound to $E_{2}$ ) without affecting the reaction catalyzed by the enzyme 2 (which is bound to $E_{1}$ ). In other words, for this enzyme complex that catalyzes a free diffusion mechanism, Eq. 36 is not fulfilled. Then, the sum of the apparent control coefficients will exceed unity for the channelled enzymes (Fig. 2) and will be equal to unity for the complexed enzymes with free diffusion (Fig. 7). We conclude, that depending on inhibitor type the sum of the apparent control coefficients determined by inhibitor titration can be used for experimental discrimination between channelling and a free diffusion mechanism [47].

The availability of inhibitors with the properties described above is rare. As an alternative to inhibitor titration an irreversible modification of the enzyme catalytic site could be performed in a separate sample (by chemical or genetic methods). Such a modification should suppress the enzyme catalytic activity only, leaving unchanged the ability of the enzyme to associate with other enzymes. Different amounts of the modified enzyme ( $e_{i}^{\text {mod }}$, which has lost the catalytic activity) should then be added to the system under study at the constant total concentration $\left(e_{i}^{t}\right)$ of the active and inactive enzyme:

$$
e_{i}^{m a d}+e_{i}=e_{i}^{l}
$$

The dependence of the flux $(J)$ on the amount of the modified enzyme $\left(e_{i}^{m o d}\right)$ is an analog of the titration curve with specific irreversible inhibitor of the enzyme $i$ (the concentration of $e_{i}^{\text {mod }}$ plays the role of the inhibitor concentrations). Transforming Eq. 31 for the irreversible inhibitor to this case, one finds:

$$
{ }^{a p p} C_{e_{1}}^{J}=-\frac{e_{i}^{l}}{J}\left(\frac{\partial J}{\partial e_{i}^{m o d}}\right)_{\mid e_{i}^{m o d}=0}
$$

The sum of the apparent control coefficients measured using the modification enzyme technique proves to exceed unity for the channelled pathways and to equal unity for the pathways with enzyme complexes but with a free diffusion mechanism of metabolite transfer. 


\section{Control of partly or completely channelled systems in general}

The conceptual shift underlying the approach developed here is to view any metabolic pathway as a number of "elemental' processes with (well-defined) forward and reverse rate constants. Notably, this implies that we no longer agglomerate all processes catalyzed by the enzyme into 'separate' reactions catalyzed in 'isolation' from the system. The general case of 'non-ideal' cellular pathway which includes $r$ enzyme reactions, is then treated as the network of chemical conversions where the 'metabolites' (system variables) are both free metabolites and different enzyme forms (states) that include enzyme-bound metabolites and enzyme-enzyme complexes (see Section 3 and [11]). These conversions are called the elemental processes of the system.

Let $n$ be the number of the elemental processes in a pathway (typically $n>r$ ), and $m$ be the total number of free metabolites and different enzyme forms (system variables). Obviously, there are some constraints on the variation of the concentrations $\left(x_{1}, x_{2}, \ldots, x_{m}\right)$ of these substances, and not all concentrations $\left(x_{k}\right)$ are independent. At least, there are $r$ constraints, corresponding to the moiety conservations of every enzyme ( $i$ ), which may have the following form:

$$
e_{i}=E_{i}+E_{i} S+\ldots E_{i} E_{j}+E_{i} S E_{j}+\ldots, i=1,2, \ldots, r
$$

Here instead of $x_{k}$ the chemical symbols are used for the concentrations: $E_{i}$ designates free enzyme, $E_{i} S$ designates the enzyme-substrate form, $E_{i} E_{i}$ and $E S E_{j}$ designate enzymeenzyme and enzyme-substrate-enzyme complexes, respectively. Such participants of the enzyme $i$ moiety-conserved cycle are called the enzyme $i$ forms (or states, cf. [59]). Obviously, complexes involving two enzymes $i$ and $j$ (e.g., $E_{i} E_{j}$ and $E S E$ ) enter both enzyme $i$ and enzyme $j$ forms simultaneously.

In what follows it is convenient to present the total enzyme concentration $e_{i}$ as the sum of the concentrations of the monomeric enzyme forms $\left(e_{i}^{\text {mon }}\right)$ (which may be complexed only with metabolites), and complexes ( $\left.e_{i}^{\text {comp }}\right)$ of the enzyme $i$ with any other enzyme $(j)$,

$$
e_{i}=e_{i}^{\mathrm{mon}}+\sum_{j \neq i} e_{i j}^{\mathrm{comp}}=e_{i}^{\mathrm{mon}}+e_{i}^{\mathrm{comp}}
$$

Here $e_{i}^{\text {comp }}$ is the part of the enzyme $i$ concentration complexed with all the other enzymes. For the sake of simplicity, we only consider heterodimer complexes (in particular, here we do not consider homodimers $e_{i} e_{j}$ ). The generalization for the case of several enzyme molecules is straight-forward.

A common feature of metabolic pathways is the presence of substrate moiety-conserved cycles [60], the interconversion of $\mathrm{NAD}^{+}$and $\mathrm{NADH}$ may serve as an example. In an arbitrary metabolic pathway $s$ such substrate moiety-conserved cycles may be present,

$$
T_{i}=\sum_{k=1}^{m} \gamma_{i k} x_{k}, i=1,2, \ldots, s
$$

Here $T_{i}$ designates the total concentration of $i$-th conserved substrate (not enzyme) moiety, and $\gamma_{i k}$ can in most cases be interpreted as the number of moieties of type $i$ in the metabolite $x_{k}$. Note, that $x_{k}$ entering Eq. 41 corresponds to the concentrations of both free and (for a different value of $k$ ) enzyme-bound metabolites. For a mathematically inclined reader we also note that the rank of the $m$ rows by $n$ columns stoichiometric matrix $\mathrm{N}$ of the metabolic network is equal to $m-r-s$. Eqs 39 and 41 describe $r+s$ linear dependencies between the rows of $\mathrm{N}$ (see, e.g., [14]).

Any elemental process (step) in the network under consideration is a mono- or bimolecular reaction with respect to the enzyme forms. Moreover, if any of the enzyme $j$ forms enter the left-hand side of chemical equation of the elemental process, then some other form of the same enzyme $j$ will enter the right-hand side of that chemical equation. Hence, the rate $\left(v_{i}\right)$ of every $i$-th elemental process is a homogeneous function of zero or first order with respect to the concentrations of the forms of any enzyme $j$ (the order equals 0 or 1 depending on whether enzyme forms participate in $i^{\text {th }}$ elemental process). All the elemental processes with rates depending on the enzyme $j$ forms were called $E_{j}$-dependent processes in Section 3.

Now we define $n$ parameters $\xi_{i}, i=1,2, \ldots, n$ each modulating the activity only of the $i$-th process,

$$
v_{i}\left(x, \xi_{i}\right)=\xi_{i} v_{i}(x, 1)
$$

According to Eq. 8 of Section 3 the elemental flux control coefficient of any process can be defined as following (cf. Eq. 7):

$$
C_{i}^{J}=\mathrm{d} \ln |J| \operatorname{d} \ln \xi_{i} \xi_{i}=1, i=1,2, \ldots, n
$$

Note, that for the classical 'macrodescription' of a pathway the parameter $\xi_{i}$ plays the role of the activity or the concentration of the enzyme and the control coefficient defined by Eq. 43 coincides with the 'classical' control coefficient (see Eq. 1).

\section{Relating control by enzymes to control by the elemental processes}

In section 3 for simple examples of channelled pathways we related the elemental to the classical control coefficients (see Eqs 14-17). To elucidate the particular features of the control in the general case of pathways which may involve channelling and moiety sequestrating, we apply a method of 
perturbation of the steady-state $[15,16]$. Let in the initial steady state the concentrations and parameters be perturbed as the following: (i) every concentration involved in the enzyme $i$ moiety-conserved cycle (see Eq. 39) is increased by a factor $\lambda_{i}$ :

$$
\begin{aligned}
& E_{i}\left(\lambda_{i}\right)=\lambda_{i} E_{i}, E S\left(\lambda_{i}\right)=\lambda_{i} E_{i} S, E_{i} E_{j}\left(\lambda_{i}\right)=\lambda_{i} E_{i} E_{j}, \\
& E_{i} S E_{j}\left(\lambda_{i}\right)=\lambda_{i} E_{i} S E_{j}, \ldots, i=1,2, \ldots, r
\end{aligned}
$$

(ii) parameters, $\xi_{-}$, which correspond to the rates of $E_{i}$-dependent elemental processes (in which any of the forms of the enzyme $i$ participates) are decreased by the same factor $\lambda_{i}$ :

$$
\xi_{k}\left(\lambda_{i}\right)=\xi_{k} / \lambda_{i},
$$

if $\xi_{k}$ corresponds to $E_{i}$-dependent process

Since the rates $v_{k}$ of $E_{i}$-dependent processes are homogeneous first-order functions of the concentrations of the enzyme $i$ forms (see above), all the rates in the new steady state will be equal to the initial non-perturbed rates. However, the parameters $\mathbf{e}, \mathbf{T}, \xi$ do differ between the old and the new steady state (see Eqs 44 and 45). The new values of $e_{j}$ and $T$, are:

$$
\begin{aligned}
& e_{i}\left(\lambda_{i}\right)=\lambda_{i} \cdot e_{i} ; \\
& \text { for } j \neq \mathrm{i}, e_{j}\left(\lambda_{i}\right)=e_{j}^{\mathrm{mon}}+\sum_{k \neq j / i} e_{j k}^{\mathrm{comp}}+\lambda_{i} \cdot e_{j i}^{\mathrm{comp}}=e_{j}+\left(\lambda_{i}-1\right) \cdot e_{j i}^{\mathrm{comp}} \text {; } \\
& T_{1}\left(\lambda_{i}\right)=T_{1}+\left(\lambda_{i}-1\right) \cdot T^{i_{i}}, 1=1,2, \ldots, s
\end{aligned}
$$

here $T_{l}^{*}$, designates the part of $l$-th conserved moiety bound to all forms of the enzyme $i$. Since the steady-state fluxes $(J)$ are functions of $(\mathbf{e}, \mathbf{T}, \xi)$ one can write:

$$
\begin{aligned}
0=\mathrm{d} \ln |J| / \mathrm{d} \ln \lambda_{i}= & \sum_{j=1}^{r} C_{e_{j}}^{J} \cdot \mathrm{d} \ln e_{j} / \mathrm{d} \ln \lambda_{i}+\sum_{l=1}^{s} R_{T_{l}}^{J} \cdot \mathrm{d} \ln T_{l} / \\
& d \ln \lambda_{i}+\sum_{k} C_{\mathrm{k}}^{\mathrm{j}} \cdot \mathrm{d} \operatorname{dn} \xi_{k} / \mathrm{d} \ln \lambda_{i} ;
\end{aligned}
$$

here $R_{T}^{J}$ is the flux response coefficient to a change in $l$-th substrate total $T_{l}$,

$$
R_{r_{l}}^{\prime}=\mathrm{d} \ln \mid J / \mathrm{d} \ln T_{l},
$$

and the third sum in Eq. 47 includes only $E_{i}$-dependent elemental processes (in which the enzyme $i$ forms participate). Substituting into Eq. 47 the derivatives with respect to $\ln \lambda_{i}$ calculated at $\lambda_{i}=1$ (using Eqs 44-46), we obtain;

$$
\begin{aligned}
& C_{e_{i}}^{j}+\sum_{j \neq i} C_{e_{j}}^{J} \cdot e_{j i}^{\text {comp } / e_{j}}+\sum_{i=1}^{s} R_{T_{j}}^{j} \cdot T_{i}^{e_{i}} / T_{i}= \\
& \sum C_{k}^{\prime}={ }^{i m p} C_{e_{i}}^{J}, i=1,2, \ldots, r \\
& \text { all } E_{i} \text {-dependent } \\
& \text { processes } k
\end{aligned}
$$

The right-hand side of this equation represents the impact control coefficient of the enzyme $i$ which has been introduced in Section 3, see Eq. 14. The left-hand side contains the classical enzyme's $i$ control coefficient, the control coefficients of those enzymes that are complexed by enzyme $i$, and the response coefficients to a modulation of conserved metabolites complexed by enzyme $i$. Note, that applying Eq. 48 to channelled pathways in Fig. 2 we arrive at Eq. 17 relating classical and elemental control coefficients for these simple channels.

\section{Concentration control}

To relate the classical concentration coefficients $\left(C_{e, ~}^{x_{k}}\right.$, Eq. 4) to the corresponding control coefficients of the elemental processes $\left(C_{j}^{x_{k}}\right.$, Eq. 9) one should take into account, that after perturbation of the parameters according to Eqs 45 and 46 , in the new steady state all the concentrations except the enzyme $i$ forms (see Eq. 44) will have the same values as in the initial steady state. Formally:

$$
\mathrm{d} \ln x_{k}=\delta\left(x_{k}, E_{i}\right) \cdot \mathrm{d} \ln \lambda
$$

Here $\delta\left(x_{k}, E_{i}\right)=1$, if $x_{k}$ is any of the forms of the enzyme $i$, otherwise, $\delta\left(x_{k}, E_{i}\right)=0$ (the argument $E_{i}$ signifies that any of the enzyme $i$ forms, monomeric or complexed, contains $E_{i}$ moiety).

Using the same approach as before, that is considering the steady-state concentrations as functions of the parameters $\mathbf{e}$, $\mathbf{T}, \xi$ one obtains:

$$
\begin{array}{r}
C_{e_{j}}^{x_{k}}+\sum_{i \neq i} C_{e_{j}}^{x_{k}} c_{j i}^{\text {comp } / e_{j}}+\sum_{i=1}^{s} R_{T_{l}}^{x_{k}} T_{i}^{x_{i}} / T_{l}= \\
\delta\left(x_{k}, E_{i}\right)+\sum_{\begin{array}{c}
\text { all } E_{j} \text {-dependent } \\
\text { processes } j
\end{array}}^{C_{j}^{x_{k}}=} \\
\delta\left(x_{k}, E_{i}\right)+{ }^{i m p} C_{e_{i}}^{x_{k}}, i=1,2, \ldots, r
\end{array}
$$

where $R_{T_{I}}^{x_{k}}$ is the response coefficient of the concentration $x_{k}$ to a change in $l$-th substrate total $T_{l}$,

$$
R_{T_{l}}^{x_{k}}=\mathrm{d} \ln x_{k} / \mathrm{d} \ln T_{I}
$$

General summation theorems for the flux and concentration control coefficients

Summing Eq. 48 over all $r$ enzymes and rearranging one obtains:

$$
\sum_{i=1}^{r} C_{e_{i}}^{J} \cdot\left(1+\frac{e_{i}^{c o m p}}{e_{i}}\right)=1+\sum_{\substack{\text { protein } \\ \text { interaction } \\ \text { steps } \mathrm{i}}} C_{i}^{J}-\sum_{l=1}^{s} R_{T_{l}}^{J} \cdot\left(\frac{T_{l}^{\text {mon }}+2 T_{l}^{c o m p}}{T_{l}}\right)
$$


here the sum of $\mathrm{T}_{l_{i}}^{e}$ over all enzymes $(i)$ is subdivided into two parts: $T_{l}^{\text {mon }}$ bound to the monomeric enzyme forms and $T_{l}^{\text {comp }}$ bound to the enzyme-enzyme complexes (note that $T_{t}^{\text {comp }}$ enter twice the sum of $T_{l}^{e_{i}}$ over all enzymes). The additional to unity sum in the right-hand side of Eq. 50 is taken over all 'protein interaction' steps, i.e., all the elemental processes in which two different enzyme moietics participate (either as monomeric or complexed enzymes).

The summation theorem for the concentration control coefficients is obtained by summing Eq. 14 over all $r$ enzymes of the pathway:

$$
\begin{aligned}
& \sum_{i=1}^{r} C_{\mathrm{e}_{i}}^{\mathrm{x}_{\mathrm{k}}}\left(1+\frac{e_{i}^{\text {comp }}}{e_{i}}\right)=\delta\left(x_{k}, E\right)+\sum_{\begin{array}{l}
\text { protein } \\
\text { interaction } \\
\text { steps i }
\end{array}}^{C_{i}^{x_{k}}-} \\
& \sum_{l=1}^{s} R_{T i}^{x_{k}} \cdot\left(\frac{T_{l}^{\mathrm{mon}}+2 T_{l}^{\text {comp }}}{T_{l}}\right)
\end{aligned}
$$

here $\delta\left(x_{k}, E\right)=1$, if $x_{k}$ is any of the forms of any of the pathway enzymes, otherwise, $\delta\left(x_{k}, E\right)=0$.

The equations derived above predict dramatic changes in the control properties of the enzymes in real cellular pathways when compared with the 'ideal' pathways considered by the classical metabolic control theory. In ideal pathways both sums on the left-hand sides of Eq. 48 and Eq. 49 , respectively vanish. In that case these formulas state that the control coefficient of an enzyme over the pathway flux or the metabolite concentration can be expressed as the sum of the elemental control coefficients of all steps in the reaction cycle of this enzyme (cf. Eq. 10).

In pathways with direct enzyme-enzyme interactions the first sum on the left-hand side of Eq. 48 or Eq. 49 reflects the contribution to the impact control coefficient of the enzyme $i$ of all those enzymes with which enzyme $i$ is complexed. In pathways with high enzyme concentrations and moiety conservations but without direct enzyme-enzyme interactions the first sum on the left-hand side of Eq. 48 or Eq. 49 equals zero, and the right-hand side of Eq. 48 or Eq. 49 , respectively, represents the control coefficient with respect to the enzyme rate or activity $[9,10,33]$. In this case Eqs 48 and 49 show that the control coefficient with respect to the enzyme concentration can be significantly less than the control coefficient with respect to the enzyme activity due to sequestration of metabolites. Indeed, in such cases the flux control coefficient with respect to the enzyme activity can even take negative values when its 'classical' analog is positive [10].

Eqs 50 and 51 present the summation theorems for the flux and concentration control coefficients valid now for an arbitrary pathway. In ideal pathways the sum of the flux control coefficients of the enzymes equals 1 (see Eq. 6, [2,5]), and the sum of the concentration control coefficients equals zero or unity depending on whether the control is considered over the concentration of any free metabolite (substrate) or any enzyme form. Eqs 50 and 51 show that sequestrating of moiety-conserved metabolites by binding them to the enzymes present in high concentrations can significantly decrease these sums. On the other hand, channelling of metabolites can affect the sum of the flux control coefficients in two different modes depending on the average complexed fraction of enzymes and on the control exerted by 'channelled' steps. The latter may reach as much as 1 , so in a pathway without substrate moiety-conservations and small life time of enzyme-enzyme complexes the sum of the flux control coefficients of the enzymes can reach 2 [48]. We shall illustrate this phenomenon considering the group transfer pathway which is an important aspect of cellular bioenergetics system.

\section{Example. Perfect dynamic channels: group-transfer} pathways

We address here highly organized cellular pathways involving the transfer of a chemical group through a series of proteins, i.e. group-transfer pathways (or relay pathways). Examples include the bacterial phosphotransferase system transferring a phosphate group from phosphoenolpyruvate to a sugar molecule whilst transporting the latter across the membrane [25], and the electron transport chain in mitochondrial and bacterial membranes transferring an electron. Such a group transfer pathway can be considered as a perfect dynamic channel in which a transferred group is not released into the bulk aqueous phase until it reaches the end of the reaction sequence.

Figure 8 shows the group-transfer pathway where a group $P$ is transferred between $r$ enzymes from the donor SP to the ultimate acceptor W. Each enzyme $(i)$ interacts with two adjacent enzymes $(i-1)$ and $(i+1)$, with which enzyme $i$ may form complexes, designated by $Q_{i-1}=E_{i-1} P E_{i}$ and $Q_{i}=E_{i} P E_{i+1}$. The complexes of enzymes 1 and $r$ with the 'boundary' substrates are designated as $Q_{0}=S P E_{1}$ and $Q_{r}=E_{r} P W$, respectively. Different forms of every enzyme ( $i$ ) participate in the four elemental processes numbered as $2 i-1,2 i, 2 i+1,2 i+2$ (see Fig. 8). Applying Eq. 48 to a group transfer pathway one arrives at:

$$
\begin{aligned}
& C_{e_{i-1}}^{J} \cdot \frac{Q_{i-1}}{e_{i-1}}+C_{e_{i}}^{J}+C_{e_{i-1}}^{J} \cdot \frac{Q_{i}}{e_{i+1}}=C_{2 i-1}^{J}+C_{2 i}^{J}+C_{2 i+1}^{J}+C_{2 i+2}^{J}= \\
& { }^{\text {imp }} C_{e_{i} 3}^{J}, i=2,3, \ldots, r-1
\end{aligned}
$$




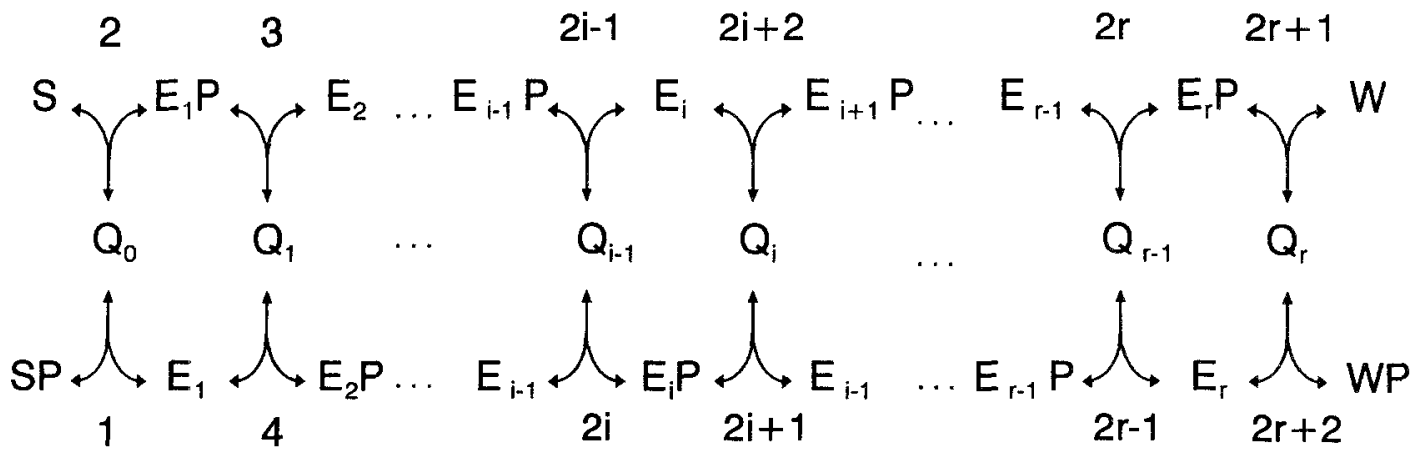

Fig. 8. Group-transfer (-relay) pathway. A group $\mathrm{P}$ is transferred between $\mathrm{r}$ enzymes from the donor SP to the ultimate acceptor W. Enzyme-enzyme complexes are referred to by $Q_{i}=E P E_{i+1}$. The complexes of enzymes 1 and $r$ with the 'boundary' substrates are designated as $Q_{0}=S P E_{1}$ and $Q_{r}=E_{r} P W$, respectively. For every enzyme $(i) E_{2}$-dependent elemental processes are numbered as $2 i-1,2 i, 2 i+1,2 i+2$.

For the initial $(i=1)$ and ultimate $(i=r)$ enzymes one obtains instead of Eq. 52:

$$
C_{e_{1}}^{J}+C_{e_{2}}^{J} \cdot \frac{Q_{1}}{e_{2}}={ }^{\mathrm{imp}} C_{e_{1}}^{J} ; \mathrm{C}_{\mathrm{e}_{\mathrm{r}-1}}^{\mathrm{J}} \cdot \frac{Q_{r-1}}{e_{r-1}}+\mathrm{C}_{\mathrm{e}_{\mathrm{r}}}^{J}={ }^{\mathrm{imp}} C_{e_{r}}^{J}
$$

As for the general case, the equations obtained allow one to express the control coefficients of the enzymes of a group transfer pathway in terms of the control coefficients of the elemental processes and the fractions of the enzymes involved in enzyme-enzyme complexes. Here we shall use Eqs 52 and 53 merely to derive the summation theorem for group transfer pathways (it can be derived from the general Eq. 50 as well). Summing these equations and adding the control exerted by the initial (S and SP) and ultimate (W, WP) substrates designated by,

$$
\begin{aligned}
& C_{e_{0}}^{J}=\frac{\mathrm{d} \ln |J|}{\mathrm{d} \ln [S]}+\frac{\mathrm{d} \ln |J|}{\mathrm{d} \ln [S P]} ; \\
& C_{e_{r+1}}^{J}=\frac{\mathrm{d} \ln |J|}{\mathrm{d} \ln [W]}+\frac{\mathrm{d} \ln |J|}{\mathrm{d} \ln [W P]}
\end{aligned}
$$

one obtains [Kholodenko and Westerhoff, submitted]:

$$
C_{e_{0}}^{J}+\sum_{i=1}^{r} C_{e_{i}}^{J} \cdot\left(1+\frac{Q_{i-1}+Q_{i}}{e_{i}}\right)+C_{e_{r+1}}^{J}=2
$$

Hence, in a group transfer pathway (perfect dynamic channel) the control exerted by the enzymes (including 'boundaries') on the pathway flux always exceeds unity. We saw, however, that in the general case of channelling it can be less than unity (see Section 3, Eq. 19).

\section{Discussion}

Metabolic control analysis and Biochemical Systems Theory have been great assets for the understanding of the control of intermediary metabolism, mitochondrial oxidative phosphorylation [reviews 39, 62]. It has founded the notion that a total flux control of $100 \%$ tends to be distributed among the enzymes participating in the pathway. It has also provided the rationale for any observed distribution of control in terms of enzyme kinetic properties. Consequently, it would seem time to apply these successful approaches experimentally to muscle and more specifically to the phosphoryl transfer from mitochondria through creatine kinase to the actinomyosin ATPase.

The bad news of this paper, i.e., that standard MCA may not be applicable to muscle energy metabolism, may have reduced the enthusiasm somewhat. Because of the possible superorganization of the high free-energy phosphate metabolism in muscle, the standard Metabolic Control Analysis may not be directly applicable. For, it assumes metabolic pathways to be ideal in the sense of consisting of chemical conversions of well-mixed metabolites by independent enzyme activities. Instead, metabolite channelling (i.e., direct transfer not involving metabolite pools) by creatine kinase isoenzymes may be of prime importance for aerobic energy metabolism [32]. And, the relative localization of contact sites between mitochondrial membranes, creatine kinase, hexokinase and the adenine nucleotide translocase may have strong implications for control.

By the terms 'superorganized' metabolism we here refer to such a level of organization that reaches beyond that of biochemical pathways inclusive of gene-expression regulation and covalent modification. Superorganization involves phenomena such as direct transfer of metabolites between enzymes, complex formation between enzymes [63], sequestration of metabolites by enzymes and restricted diffusion.

The good news of this paper is that the analogue of MCA that is able to deal with such superorganized metabolism has now been developed. And, it has turned out that such metabolism has quite distinct control properties.

For instance, the sum of the flux control coefficients by enzymes is the golden standard of $100 \%$ in ideal pathways. 
In superorganized pathways it tends to exceed 1 because of direct transfer of metabolites and it tends to be lower than 1 because of metabolite sequestration. We showed how measuring the responses of a channelled pathway to external effectors (e.g., inhibitors) can enhance the insight into regulatory properties of the pathway.

As a consequence, control studies may be used to determine the functional extent to which metabolism is organized. Early on, such a use was pioneered by Baum and colleagues [64] and Kell and colleagues in studies of possible superorganization in chemiosmotic coupling $[34,36]$.

The analysis presented here may remove an important limitation of metabolic control theory. Moreover, it provides new definitions that should facilitate the quantitative characterization of metabolite channelling, without taking the system apart. The theory developed here also allows one to analyze implications of channelling for the regulation of cellular metabolism.

More in particular, determining for group transfer pathways the sum of the flux control coefficients of enzymes and in particular its deviation from 1 and 2 may serve as a measure of the extent of complexation of the proteins in the pathway. This may be one of the few methods by which such complexation can be demonstrated in cases where cell disruption leads to dissociation of such complexes [22].

The present study also suggests that in case of a dynamic organization of the components of the electron transfer chain in membranes [65], the sum of the control coefficients on the flux of reducing equivalents should equal two [cf. 48], whereas in the case of a static organization [cf. 66] it should equal unity. Existing data lack sufficient resolution to decide.

Mitochondrial creatine kinase is an attractive alternative candidate to prove that metabolite channelling by dynamic enzyme complexes occurs. For, various studies suggest that the adenine nucleotide translocase, mitochondrial creatine kinase of the intermembrane space and porin of the outer mitochondrial membrane channel high-energy phosphates from the matrix to the cytosol $[23,32]$.

A second asset of the new control theory is that it suggests that the muscle system might exhibit extra regulation precisely because of its superorganization properties. In 'simple' metabolic pathways the response to an external signal is readily described in terms of the effect of the signal on its receptor enzyme and the control exerted by that enzyme. We have shown that in the response of 'channelled' pathways to such a signal, additional terms appear that reflect the direct enzyme-enzyme interactions [cf. 67]. They tend to enhance the responsiveness of the pathway. The normalized value of the response was called the signal transduction coefficient. We showed that in channelled pathways these coefficients are usually larger than in corresponding non-channelled (simple) pathways.

We conclude that the time is ripe for a quantitative under- standing of control and regulation of muscle physiology [see for instance 68]. The control theory developed here may serve to suggest an approach. It will certainly help in understanding why control in such systems is aberrant from that in ideal pathways.

\section{Acknowledgements}

This work was supported by the Russian University Grant, by the Spanish Advice Commission for the Scientific and Technical Research (CICYT, PB 92-0852) and by the Netherlands Organization for Scientific Research.

\section{References}

1. Higgins JJ: Dynamics and control in cellular reactions. In: B Chance, RW Estabrook, JR Williamson (eds) Control of Energy Metabolism. Academic Press, New York, 1965, pp 13-46

2. Kacser H, Burns JA: The control of the flux. In: DD Davies (ed.) Rate Control of Biological Processes. Cambridge Univ. Press, London, 1973, pp 65-104

3. Kacser H, Bums JA: Molecular democracy: who shares the controls? Biochem Soc Trans 7: 1149-1160, 1979

4. Savageau MA: The behavior of intact biochemical control systems. Current Topics in Cellular Regulation 6: 63-130, 1972

5. Heinrich R, Rapoport TA: A linear steady-state treatment of enzymatic chains. General properties, control and effector strength. Europ $\mathbf{J}$ Biochem 42: 89-95, 1974

6. Heinrich R, Rapoport TA: Mathematical analysis of multi-enzyme systems: II. Steady state and transient control. Bio Systems 7: $130-136,1975$

7. Burns JA, Cornish-Bowden A, Grown AK, Heinrich R, Kacser H, Porteous JW, Rapoport SM, Rapoport T, Stucki JW, Tager JM, Wanders RJA, Westerhoff HV: Control analysis of metabolic systems, Trends Biochem Sci 10: 16, 1985

8. Kacser H, Sauro HM, Acerenza L: Enzyme-enzyme interactions and control analysis. The case of non-additivity: monomer-oligomer associations. Eur J Biochem 187: 481-491, 1990

9. Fell DA, Sauro HM: Metabolic control analysis. The effects of high enzyme concentrations. Eur J Biochem 192: 183-187, 1990

10. Kholodenko BN, Lyubarev AE, Kurganov Bl: Control of metabolic flux in a system with high enzyme concentrations and moietyconserved cycles. The sum of the flux control coefficients can drop significantly below unity. Eur J Biochem 210: 147-153, 1992

11. Kholodenko BN, Westerhoff HV: Metabolic channelling and control of the flux. FEBS Lett 320; 71-74, 1993

12. Heinrich R, Rapoport SM, Rapoport TA: Metabolic regulation and mathematical models. Prog Biophys Mol Biol 32: 1-83, 1977

13. Groen AK, Wanders RJA, Westerhoff HV, Van der Meer R, Tager JM: Quantification of the contribution of various steps to the control of mitochondrial respiration. J Biol Chem 257: 2754-2757, 1982

14. Reder C: Metabolic control theory: a structural approach. J Theor Biol 135: 175-201, 1988

15. Kholodenko BN: Control of molecular transformations in multienzyme systems: quantitative theory of metabolic regulation. Mol Biol (USSR) 22: 1238-1256, 1988 (English trans), 22: 990-1005)

16. Fell DA, Sauro HM: Metabolic control and its analysis: additional relationships between elasticities and control coefficients. Eur J 
Biochem 148: 555-561, 1985

17. Cascante M, Franco R, Canela EI: Use of implicit methods from general sensitivity theory to develop a systematic approach to metabolic control. II. Complex systems. Mathem Biosci 94: 289-309, 1989

18. Westerhoff HV, Hofmeyr J-H S, Kholodenko BN: Getting to the inside of cells using metabolic control analysis. Biophys Chem. 50: 273-283, 1994

19. Srere PA: Complexes of sequential metabolic enzymes. Ann Rev Bioch 56: 89-124, 1987

20. Srere PA, Ovadi J: Enzyme-enzyme interactions and their metabolic role. FEBS Lett 268: 360-364, 1989

21. Welch GR, Clegg JS (eds) The organization of cell metabolism. New York, Plenum Press, 1986

22. Clegg JS: Cellular infrastructure and metabolic organization. In: Current Topics in Cellular Regulation 33: 3-14, 1992

23. Wallimann T, Wys M, Brdiczka D, Nicolay K, Eppenberger HM: Intracellular compartmentation, structure and function of creatine kinase isoenzymes in tissues with high and fluctuating energy demands: the 'phosphocreatine circuit' for cellular energy homeostasis. Biochemical J 281: 21-40, 1992

24. Srivastava DK, Bernard SA: Biophysical chemistry of metabolic reaction sequences in concentrated enzyme solution and in the cell. Ann Rev of Biophys and Biophys Chem 16:175-204, 1987

25. Postma PW, Lengeler JW: Phosphoenolpyruvate:carbohydrate phosphotransferase system of bacteria. Microbiol Rev 49: 232-269

26. Skulachev VP: Membrane Bioenergetics. Springer, Berlin, 1988

27. Kell DB, Westerhoff HV: Catalytic facilitation and membrane bioenergetics. In: GR Welch (ed.) Organized Multienzyme Systems. Academic Press, New York, 1985, pp 63-138

28. Gellerich FN, Khuchua ZA, Kuznetsov AV: Influence of the mitochondrial outer membrane and the binding of creatine kinase to the mitochondrial inner membrane on the compartmentation of adenine nucleotides in the intermembrane space of rat liver mitochondria. Biochim Biophys Acta 1140: 327-334, 1993

29. Saks VA, Rosenstraukh LV, Smirnov VN, Chazov EI: Role of creatine phosphokinase in cellular function and metabolism. Can J Physiol Pharmacol 56: 691-706, 1978

30. Saks VA, Vasileva E, Belicova Yu O, Kuznetsov AV, Lyapina S, Petrova L, Perov NA: Retarded diffusion of ADP in cardiomyocites: possible role of mitochondrial outer membrane and the creatine kinase in cellular regulation of oxidative phosphorylation. Biochim Biophys Acta 1144: 134-148, 1993

31. Gellerich FN: The role of adenylate kinase in dynamic compartmentation of adenine nucleotides in the intermembrane space of rat heart mitochondria. FEBS Lett 297: 55-58, 1992

32. Wyss $M$, Wallimann $T$ : Metabolite channelling in aerobic energy metabolism. J Theor Biol 158: 129-132, 1992

33. Sauro HM, Kacser $\mathrm{H}$ : Enzyme-enzyme interactions and control analysis. The case of non-independence: heterologous associations. Eur J Biochem 187: 493-500, 1990

34. Westerhoff HV, Kell DB: A control theoretical analysis of inhibitor titration assays of metabolic channelling. Comm Mol Cell Biophys 5: $57-107,1988$

35. Easterby JS: Temporal analysis of the transition between steady states. In: A Cornish-Bowden, ML Cardenas (eds) Control of Metabolic Processes. Plenum Press, New York, 1990, pp 290-291

36. Kell DB, Westerhoff HV: In: PA Srere, ME Jones, CK Mathews (eds) Structural and Organizational Aspects of Metabolic Regulation. Wiley-Liss, New York, 1990, pp 273-289

37. Schuster S, Heinrich R: The definitions of metabolic control analysis revisited. BioSystems 27: 1-15, 1992

38. Westerhoff HV, Van Dam K: Thermodynamics and Control of
Biological Free-Energy Transduction. Elsevier, Amsterdam, 1987

39. Giersch C: Control analysis of metabolic networks. 1. Homogeneous functions and the summation theorems for control coefficients. Eur $\mathrm{J}$ Biochem 174: 509-513, 1988

40. Kholodenko BN, Westerhoff HV: Sum of the flux control coefficients: What is it equal to in different systems? In: Schuster S, Rigoulet M, Ouhabi R, Mazat J (eds) Modern Trends in BiothermoKinetics. Plenum, New York, London, 1994, pp 205-210

41. Yanofsky C: Tryptophan synthetase of E. coli: a multifunctional, multicomponent enzyme. Biochim Biophys Acta 1000: 133-137, 1989

42. Gutfreund H, Chock PB: Substrate channelling among glycolytic enzymes - fact or fiction. J Theor Biol 152: 117-121, 1991

43. Wu X, Gutfreund H, Lakatos S, Chock PB: Substrate channelling in glycolysis: a phantom phenomenon. Proc Natl Acad Sci USA 88: $497-501,1991$

44. Brooks SPJ, Storey KB: Re-evaluation of the glycerol-3-phosphate dehydrogenase/L-lactate dehydrogenase enzyme system. Evidence against the direct transfer of NADH between active sites. Biochem $J$ 278: 875-881, 1991

45. Smolen P, Keizer J: Kinetics and thermodynamics of metabolite transfer between enzymes. Biophys Chem 38: 241-263, 1990

46. Ovady J: Physiological significance of metabolic channelling. J Theor Biol 152: 1-22, 1991

47. Kholodenko BN: Control theory of 'non-classical' enzyme systems and methods for the study of metabolic channelling. Biochemistry (USSR) English transl 58: 325-337, 1993

48. Van Dam K, Van der Vlag J, Kholodenko BN, Westerhoff HV: The sum of the control coefficients of all enzymes on the flux through a group-transfer pathway can be as high as two. Eur J Biochem 212: $791-799,1993$

49. Kholodenko BN: How do external parameters control fluxes and concentrations of metabolites? An additional relationship in the theory of metabolic control. FEBS Lett 232: 383-386, 1988

50. Torres NV, Mateo F, Melendez-Hevia E, Kacser H: Kinetics of metabolic pathways. A system in vitro to study the control of flux. Biochem J 234: 169-174, 1986

51. Flint HJ, Tateson RW, Barthelmess IB, Porteous DJ, Donachie WD, Kacser H: Control of the flux in the arginine pathway of Neurospora crassa. Modulations of enzyme activity and concentrations. Biochem J 200: $231-246,1981$

52. Walsh K, Koshland DE Jr: Proc Natl Acad Sci USA 82: 3577-3581, 1985

53. Jensen PR, Westerhoff HV, Michelsen $\mathrm{O}$ : Excess capacity of $\mathrm{H}^{+}$ATPase and inverse respiratory control in E. coli. EMBO J 12: 1277-1282, 1993

54. Groen AK, Wanders RJA, Westerhoff HV, Van der Meer R, Tager JM: Quantification of the contribution of various steps to the control of mitochondrial respiration. J Biol Chem 257: 2754-2757, 1982

55. Kholodenko B, Zilinskiene V, Borutaite V, Ivanovene L, Toleikis A, Praskevicius A: The role of adenine nucleotide translocator in regulation of oxidative phosphorylation in heart mitochondria. FEBS Lett 223: 247-250, 1987

56. Rigoulet M, Averet N, Mazat J-P, Gurin B, Cohadon F: Redistribution of the flux-control coefficients in mitochondrial oxidative phosphorylation in the course of brain edema. Biochim Biophys Acta 932: 116-123, 1988

57. Kelety T: Basic Enzyme Kinetics. Academiai Kiado, Budapest, 1986

58. Cornish-Bowden A: Principles of Enzyme Kinetics. Butterworth, London, 1976

59. Hill TL: Free Energy Transduction in Biology. Academic Press, New York, 1977

60. Hofmeyr J-HS, Kacser H, Van der Merwe KJ: Metabolic control analysis of moiety conserved cycles. Eur J Biochem 155: 631-641, 
1986

61. Kholodenko BN: Metabolic control theory. New relationships for determining control coefficients of enzymes and response coefficients of system variables. J Nonlinear Biol 1: 107-126, 1991

62. Fell DA: Metabolic control analysis: a survey of its theoretical and experimental development. Biochem J 286: 313-330, 1992

63. Kurganov BI: Allosteric Enzymes: Kinetic Behaviour. John Wiley \& Son, 1978

64. Baum H: In: S Fleischer, Y Hatefi, DM McLennan, A Tzagoloff (eds) Molecular Biology of Membranes. Plenum, New York, pp 243-262, 1978

65. Hackenbrock CR, Gupte SS: In: JJ Lemasters, CR Hackenbrock, RG Thurman, HV Westerhoff (eds) Integration of Mitochondrial Function.
Plenum Press, New York, 1988, pp 15-22

66. Ferguson-Miller S, Rajarathnam K, Kochman J, Schindler M: In: JJ Lemasters, CR Hackenbrock, RG Thurman, HV Westerhoff (eds) Integration of Mitochondrial Function. Plenum Press, New York, 1988, pp 23-32

67. Kholodenko BN, Demin O, Westerhoff HV: 'Channelled' pathways can be more sensitive to specific regulatory signals. FEBS Lett 320: $75-78,1993$

68. Hak JB, Van Beek JHGM, van Wije MH, Eijgelshoven MHJ, Westerhoff N: Reduced cardiac ATP-synthetic capacity slows metabolic regulation and reduces contractility. Circ Res, in press, 1994 